\title{
On Hyers-Ulam stability of a multi-order boundary value problems via Riemann-Liouville derivatives and integrals
}

\author{
Salim Ben Chikh', Abdelkader Amara', Sina Etemad ${ }^{2}$ and Shahram Rezapour ${ }^{3,4,5^{*}}$ (1)
}

"Correspondence:
shahramrezapour@duytan.edu.vn;
sh.rezapour@mail.cmuh.org.tw;
sh.rezapour@azaruniv.ac.ir;
rezapourshahram@yahoo.ca
${ }^{3}$ Institute of Research and
Development, Duy Tan University,
Da Nang 550000, Vietnam
${ }^{4}$ Faculty of Natural Sciences, Duy
Tan University, Da Nang 550000,
Vietnam
Full list of author information is
available at the end of the article

*Correspondence:

rezapourshahram@yahoo.ca

${ }^{3}$ Institute of Research and

Da Nang 550000, Vietnam

${ }^{4}$ Faculty of Natural Sciences, Duy

Full list of author information is

\begin{abstract}
In this research paper, we introduce a general structure of a fractional boundary value problem in which a 2-term fractional differential equation has a fractional bi-order setting of Riemann-Liouville type. Moreover, we consider the boundary conditions of the proposed problem as mixed Riemann-Liouville integro-derivative conditions with four different orders which cover many special cases studied before. In the first step, we investigate the existence and uniqueness of solutions for the given multi-order boundary value problem, and then the Hyers-Ulam stability is another notion in this regard which we study. Finally, we provide two illustrative examples to support our theoretical findings.
\end{abstract}

MSC: Primary 34A08; secondary 34A12

Keywords: Boundary value problem; Hyers-Ulam stability; Multi-order fractional differential equation; Riemann-Liouville derivative

\section{Introduction and preliminaries}

Fractional differential problems have drawn much interest in recent years owing to their extensive utilization in different branches of science such as engineering, mechanics, potential theory, biology, chemistry, etc. (see [1-11]). Many researchers helped in developments on the existence and uniqueness results of fractional differential equations [12-26]. Stability is a notion in physics, and most phenomena include the concept. In fact, stability of physical phenomena has an old history, and one can find a lot of works in the literature not only in the last century but also before it [27-36]. During recent decades, considerable attention has been given to the study of the Hyers-Ulam stability of functional differential and integral equations [37-54].

In 2016, Niyom et al. studied the boundary value problem via four-order fractional Riemann-Liouville derivatives

$$
\left\{\begin{array}{l}
\lambda \mathcal{D}^{k}(u(t))+(1-\lambda) \mathcal{D}^{\theta}(u(t))=\hat{\Upsilon}(t, u(t)) \quad(t \in[0, T], k \in(1,2]), \\
u(0)=0, \quad \mu_{1} \mathcal{D}^{\gamma_{1}} u(T)+\left(1-\mu_{1}\right) \mathcal{D}^{\gamma_{2}} u(T)=\delta_{1},
\end{array}\right.
$$

(c) The Author(s) 2020. This article is licensed under a Creative Commons Attribution 4.0 International License, which permits use, sharing, adaptation, distribution and reproduction in any medium or format, as long as you give appropriate credit to the original author(s) and the source, provide a link to the Creative Commons licence, and indicate if changes were made. The images or other third party material in this article are included in the article's Creative Commons licence, unless indicated otherwise in a credit line to the material. If material is not included in the article's Creative Commons licence and your intended use is not permitted by statutory regulation or exceeds the permitted use, you will need to obtain permission directly from the copyright holder. To view a copy of this licence, visit http://creativecommons.org/licenses/by/4.0/. 
under some conditions [55]. In 2017, Ntouyas et al. reviewed a boundary value problem via multiple orders of fractional derivatives and integrals

$$
\left\{\begin{array}{l}
\lambda \mathcal{D}^{k}(u(t))+(1-\lambda) \mathcal{D}^{\theta}(u(t))=\hat{\Upsilon}(t, u(t)) \quad(t \in[0, T], k \in(1,2]), \\
u(0)=0, \quad \mu_{2} \mathcal{I}^{q_{1}} u(T)+\left(1-\mu_{2}\right) \mathcal{I}^{q_{2}} u(T)=\delta_{2}
\end{array}\right.
$$

under some conditions [15]. In 2018, Xu et al. investigated the existence of solutions and Hyers-Ulam stability for the fractional differential equations

$$
\left\{\begin{array}{l}
\lambda \mathcal{D}^{k}(u(t))+\mathcal{D}^{\theta}(u(t))=\hat{\Upsilon}(t, u(t)) \quad(t \in[0, T], k \in(1,2]), \\
u(0)=0, \quad \mu_{1} \mathcal{D}^{\gamma_{1}} u(T)+\mathcal{I}^{q_{2}} u(v)=\delta_{2},
\end{array}\right.
$$

under some conditions [39]. They considered two-term class of three-point boundary value problems with Riemann-Liouville fractional derivatives and integrals [39].

Now, by using and mixing the idea of the above-mentioned works, we consider a new category of boundary value problem including multi-order Riemann-Liouville fractional equation supplemented with different linear integro-derivative boundary conditions as follows:

$$
\left\{\begin{array}{l}
\lambda \mathcal{D}^{k}(u(t))+(1-\lambda) \mathcal{D}^{\theta}(u(t))=\hat{\Upsilon}(t, u(t)) \quad(t \in[0, T], k \in[2,3)) \\
u(0)=0, \quad \mu_{1} \mathcal{D}^{\gamma_{1}} u(T)+\left(1-\mu_{1}\right) \mathcal{D}^{\gamma_{2}} u(T)=\delta_{1} \\
\mu_{2} \mathcal{I}^{q_{1}} u(T)+\left(1-\mu_{2}\right) \mathcal{I}^{q_{2}} u(T)=\delta_{2}
\end{array}\right.
$$

where $2<\theta<k, 0<\lambda, \mu_{1}, \mu_{2} \leq 1,0 \leq \gamma_{1}, \gamma_{2}<k-\theta, q_{1}, q_{2} \in \mathbb{R}^{+}, \mathcal{D}^{\beta}$ is the RiemannLiouville fractional derivative of order $\beta \in\left\{k, \theta, \gamma_{1}, \gamma_{2}\right\}, \mathcal{I}^{\eta}$ denotes the Riemann-Liouville fractional integral of order $\eta \in\left\{q_{1}, q_{2}\right\}$, and the map $\hat{\Upsilon}:[0, T] \times \mathbb{R} \rightarrow \mathbb{R}$ is continuous.

As many researchers would like to investigate the stability notion of different boundary value problems, this can be a motivation for us to study the stability of complicated systems supplemented with general boundary conditions. Hence more precisely, our main goal in the present manuscript is to obtain some existence criteria of the solutions for a new general boundary value problem including 2-term fractional differential equation (4) which contains multi-order Riemann-Liouville fractional derivatives and integrals. To fulfil this aim, we use the well-known standard fixed point theorems. Also, in the sequel, we investigate the Hyers-Ulam stability of the proposed problem (4) in the special case $\mu_{1}=1$ and $\mu_{2}=1$. Finally, we present two illustrative examples to show the validity of our theoretical findings. We believe that such proposed boundary value problem is general, and it involves many fractional dynamical systems as special cases in physics and other applied sciences.

\section{Preliminaries}

Now, let us provide some basic notions. It is known that the Riemann-Liouville fractional integral of order $\eta$ of a real-valued function $g:(0, \infty) \rightarrow \mathbb{R}$ is defined by $\mathcal{I}^{\eta} g(t)=$ $\int_{0}^{t} \frac{(t-s)^{\eta-1}}{\Gamma(\eta)} g(s) d s$, provided the right-hand side is point-wise defined on $(0, \infty)$, where $\Gamma$ is the gamma function [1]. The Riemann-Liouville fractional derivative of order $k$ of a func- 
tion $g:(0, \infty) \rightarrow \mathbb{R}$ is defined by $\mathcal{D}^{\beta} g(t)=\frac{1}{\Gamma(n-\beta)}\left(\frac{d}{d t}\right)^{n} \int_{0}^{t} \frac{g(s)}{(t-s)^{\beta-n+1}} d s$, where $n=[\beta]+1,[\beta]$ denotes the integer part of real number $\beta$ provided the right-hand side is point-wise defined on $(0, \infty)[1]$. We need the next results.

Lemma $1([1,5])$ Let $k>0$ and $u \in C(0,1)$, where $C(0,1)$ stands for the space of all continuous real-valued functions defined on $(0,1)$. Then the fractional differential equation $\mathcal{D}^{k} u(t)=0$ has a general solution $u(t)=C_{1} t^{k-1}+C_{1} t^{k-2}+\cdots+C_{1} t^{k-n}$, where $n-1 \leq k<n$ and $C_{1}, \ldots, C_{n}$ are some real constants.

Lemma 2 ([1]) Let $k>0$ and $u \in C(0,1)$. Then we have

$$
\mathcal{I}^{k} \mathcal{D}^{k} u(t)=u(t)+C_{1} t^{k-1}+C_{1} t^{k-2}+\cdots+C_{1} t^{k-n},
$$

where $n-1 \leq k<n$ and $C_{1}, \ldots, C_{n}$ are some real constants.

Theorem 3 (Krasnoselskii's fixed point theorem, [56]) Let $\mathcal{M}$ be a closed, bounded, convex, and nonempty subset of a Banach space $\mathcal{X}$. Assume that $\mathcal{A}_{1}$ and $\mathcal{A}_{2}$ are two operators on $\mathcal{M}$ such that

(a) $\mathcal{A}_{1} u+\mathcal{A}_{2} w \in \mathcal{M}$ for all $u, w \in \mathcal{M}$,

(b) $\mathcal{A}_{1}$ is compact and continuous,

(c) $\mathcal{A}_{2}$ is a contraction.

Then there exists $z \in \mathcal{M}$ such that $z=\mathcal{A}_{1} z+\mathcal{A}_{2} z$.

Theorem 4 (Leray-Schauder's nonlinear alternative, [57]) Let $\mathcal{X}$ be a Banach space, $\mathcal{B}$ be a closed, convex subset of $\mathcal{X}, \mathcal{U}$ be an open subset of $\mathcal{B}$, and $0 \in \mathcal{U}$. Assume that $\mathcal{P}: \overline{\mathcal{U}} \rightarrow \mathcal{B}$ is a continuous and compact map. Then either

(a) $\mathcal{P}$ has a fixed point in $\overline{\mathcal{U}}$, or

(b) there is $u \in \partial \mathcal{U}$ (the boundary of $\mathcal{U}$ ) and $\tau \in(0,1)$ with $u=\tau \mathcal{P}(u)$.

Lemma 5 ([39]) Let $\alpha>0, a>0, g(t, s)$ be a nonnegative continuous bounded function defined on $[0, T] \times[0, T]$ and nondecreasing with respect to the first variable and nonincreasing with respect to the second variable. Assume that $u(t)$ is nonnegative and integrable on $[0, T]$ with $u(t) \leq a+\int_{0}^{t} g(t, s)(t-s)^{\alpha-1} u(s) d s$ for $t \in[0, T]$. Then we have

$$
u(t) \leq a+a \int_{0}^{t} \sum_{n=1}^{\infty} \frac{(g(t, s) \Gamma(\alpha))^{n}}{\Gamma(n \alpha)}(t-s)^{n \alpha-1} d s
$$

Theorem 6 (Banach contraction principle, [57]) Let $\mathcal{X}$ be a Banach space and $\mathcal{P}: \mathcal{X} \rightarrow \mathcal{X}$ be a contraction. Then $\mathcal{P}$ has a unique fixed point.

\section{Some existence results}

Let $T>0, J=[0, T]$, and $\mathcal{C}=C(J, \mathbb{R})$ be the Banach space of continuous mappings with the sup norm $\|u\|=\sup _{t \in J}|u(t)|$. We first provide our key result. 
Lemma 7 A map $u_{0}$ is a solution for boundary value problem (4) if and only if $u_{0}$ is a solution for the integral equation

$$
\begin{aligned}
u(t)= & \frac{\lambda-1}{\lambda \Gamma(k-\theta)} \int_{0}^{t}(t-s)^{k-\theta-1} u(s) d s+\frac{1}{\lambda \Gamma(k)} \int_{0}^{t}(t-s)^{k-1} \hat{\Upsilon}(t, u(t)) d s \\
& +\frac{t^{k-1}}{\Theta} \times\left[\frac{\mu_{1} \Lambda_{4}(\lambda-1)}{\lambda} \mathcal{I}^{k-\theta-\gamma_{1}} u(T)-\frac{\Lambda_{2} \mu_{2}(\lambda-1)}{\lambda} \mathcal{I}^{k-\theta+q_{1}} u(T)\right. \\
& +\frac{\Lambda_{4}\left(1-\mu_{1}\right)(\lambda-1)}{\lambda} \mathcal{I}^{k-\theta-\gamma_{2}} u(T)-\frac{\Lambda_{2}\left(1-\mu_{2}\right)(\lambda-1)}{\lambda} \mathcal{I}^{k-\theta+q_{2}} u(T) \\
& +\frac{\Lambda_{4} \mu_{1}}{\lambda} \mathcal{I}^{k-\gamma_{1}} \hat{\Upsilon}(T, u(T))-\frac{\Lambda_{2} \mu_{2}}{\lambda} \mathcal{I}^{k+q_{1}} \hat{\Upsilon}(T, u(T))+\Lambda_{2} \delta_{2}-\Lambda_{4} \delta_{1} \\
& \left.+\frac{\Lambda_{4}\left(1-\mu_{1}\right)}{\lambda} \mathcal{I}^{k-\gamma_{2}} \hat{\Upsilon}(T, u(T))-\frac{\Lambda_{2}\left(1-\mu_{2}\right)}{\lambda} \mathcal{I}^{k+q_{2}} \hat{\Upsilon}(T, u(T))\right] \\
& -\frac{t^{k-2}}{\Theta}\left[\frac{\mu_{1} \Lambda_{3}(\lambda-1)}{\lambda} \mathcal{I}^{k-\theta-\gamma_{1}} u(T)-\frac{\Lambda_{1} \mu_{2}(\lambda-1)}{\lambda} \mathcal{I}^{k-\theta+q_{1}} u(T)\right. \\
& +\frac{\Lambda_{3}\left(1-\mu_{1}\right)(\lambda-1)}{\lambda} \mathcal{I}^{k-\theta-\gamma_{2}} u(T)-\frac{\Lambda_{1}\left(1-\mu_{2}\right)(\lambda-1)}{\lambda} \mathcal{I}^{k-\theta+q_{2}} u(T) \\
& +\frac{\Lambda_{3} \mu_{1}}{\lambda} \mathcal{I}^{k-\gamma_{1}} \hat{\Upsilon}(T, u(T))-\frac{\Lambda_{1} \mu_{2}}{\lambda} \mathcal{I}^{k+q_{1}} \hat{\Upsilon}(T, u(T))+\Lambda_{1} \delta_{2}-\Lambda_{3} \delta_{1} \\
& \left.+\frac{\Lambda_{3}\left(1-\mu_{1}\right)}{\lambda} \mathcal{I}^{k-\gamma_{2}} \hat{\Upsilon}(T, u(T))-\frac{\Lambda_{1}\left(1-\mu_{2}\right)}{\lambda} \mathcal{I}^{k+q_{2}} \hat{\Upsilon}(T, u(T))\right]
\end{aligned}
$$

where

$$
\begin{aligned}
& \Lambda_{1}=\frac{\mu_{1} \Gamma(k)}{\Gamma\left(k-\gamma_{1}\right)} T^{k-\gamma_{1}-1}+\frac{\left(1-\mu_{1}\right) \Gamma(k)}{\Gamma\left(k-\gamma_{2}\right)} T^{k-\gamma_{2}-1}, \\
& \Lambda_{2}=\frac{\mu_{1} \Gamma(k-1)}{\Gamma\left(k-\gamma_{1}-1\right)} T^{k-\gamma_{1}-2}+\frac{\left(1-\mu_{1}\right) \Gamma(k-1)}{\Gamma\left(k-\gamma_{2}-1\right)} T^{k-\gamma_{2}-2}, \\
& \Lambda_{3}=\frac{\mu_{2} \Gamma(k)}{\Gamma\left(k+q_{1}\right)} T^{k+q_{1}-1}+\frac{\left(1-\mu_{2}\right) \Gamma(k)}{\Gamma\left(k+q_{2}\right)} T^{k+q_{2}-1}, \\
& \Lambda_{4}=\frac{\mu_{2} \Gamma(k-1)}{\Gamma\left(k+q_{1}-1\right)} T^{k+q_{1}-2}+\frac{\left(1-\mu_{2}\right) \Gamma(k-1)}{\Gamma\left(k+q_{2}-1\right)} T^{k+q_{2}-2}, \\
& \Theta=\Lambda_{3} \Lambda_{2}-\Lambda_{1} \Lambda_{4} .
\end{aligned}
$$

Proof First, assume that $u_{0}$ is a solution for problem (4). Then we have

$$
\mathcal{D}^{k} u_{0}(t)=\frac{\lambda-1}{\lambda} \mathcal{D}^{k} u_{0}(t)+\frac{1}{\lambda} \hat{\Upsilon}\left(t, u_{0}(t)\right)
$$

By taking the Riemann-Liouville fractional integral of order $k$ from both sides of equation (7), we obtain

$$
\begin{aligned}
u_{0}(t)= & \frac{\lambda-1}{\lambda \Gamma(k-\theta)} \int_{0}^{t}(t-s)^{k-\theta-1} u_{0}(s) d s+\frac{1}{\lambda \Gamma(k)} \int_{0}^{t}(t-s)^{k-1} \hat{\Upsilon}\left(s, u_{0}(s)\right) d s \\
& +C_{1} t^{k-1}+C_{2} t^{k-2}+C_{3} t^{k-3}
\end{aligned}
$$


for some real constants $C_{1}, C_{2}$, and $C_{3}$. For $2<k<3$, the first boundary condition of (4) implies that $C_{3}=0$. Hence,

$$
u_{0}(t)=\frac{\lambda-1}{\lambda} \mathcal{I}^{k-\theta} u_{0}(t)+\frac{1}{\lambda} \mathcal{I}^{k} \hat{\Upsilon}\left(t, u_{0}(t)\right)+C_{1} t^{k-1}+C_{2} t^{k-2}
$$

By using the Riemann-Liouville fractional derivative and integral of order $\alpha$ and $\beta$ respectively with $\alpha \in\left\{\gamma_{1}, \gamma_{2}\right\}, \beta \in\left\{q_{1}, q_{1}\right\}, 0<\alpha<k-\theta$, and $2<\theta<k$, we get

$$
\begin{aligned}
\mathcal{D}^{\alpha} u_{0}(t)= & \frac{\lambda-1}{\lambda \Gamma(k-\theta-\alpha)} \int_{0}^{t}(t-s)^{k-\theta-\alpha-1} u_{0}(s) d s+C_{1} \frac{\Gamma(k)}{\Gamma(k-\alpha)} t^{k-\alpha-1} \\
& +\frac{1}{\lambda \Gamma(k-\alpha)} \int_{0}^{t}(t-s)^{k-\alpha-1} \hat{\Upsilon}\left(s, u_{0}(s)\right) d s+C_{2} \frac{\Gamma(k-1)}{\Gamma(k-\alpha-1)} t^{k-\alpha-2}
\end{aligned}
$$

and

$$
\begin{aligned}
\mathcal{I}^{\beta} u_{0}(t)= & \frac{\lambda-1}{\lambda \Gamma(k-\theta+\beta)} \int_{0}^{t}(t-s)^{k-\theta+\beta-1} u_{0}(s) d s+C_{1} \frac{\Gamma(\alpha)}{\Gamma(k+\beta)} t^{k+\beta-1} \\
& +\frac{1}{\lambda \Gamma(k+\beta)} \int_{0}^{t}(t-s)^{k+\beta-1} \hat{\Upsilon}\left(s, u_{0}(s)\right) d s+C_{2} \frac{\Gamma(k-1)}{\Gamma(k+\beta-1)} t^{k+\beta-2} .
\end{aligned}
$$

By replacing the values $\alpha=\gamma_{1}, \alpha=\gamma_{2}, \beta=q_{1}$, and $\beta=q_{2}$ and using the second condition of (4), we get

$$
\begin{aligned}
& \frac{\mu_{1}(\lambda-1)}{\lambda \Gamma\left(k-\theta-\gamma_{1}\right)} \int_{0}^{T}(T-s)^{k-\theta-\gamma_{1}-1} u_{0}(s) d s \\
& \quad+\frac{\left(1-\mu_{1}\right)(\lambda-1)}{\lambda \Gamma\left(k-\theta-\gamma_{2}\right)} \int_{0}^{T}(T-s)^{k-\theta-\gamma_{2}-1} u_{0}(s) d s \\
& \quad+\frac{\mu_{1}}{\lambda \Gamma\left(k-\gamma_{1}\right)} \int_{0}^{T}(T-s)^{k-\gamma_{1}-1} \hat{\Upsilon}\left(s, u_{0}(s)\right) d s \\
& \quad+\frac{\left(1-\mu_{1}\right)}{\lambda \Gamma\left(k-\gamma_{2}\right)} \int_{0}^{T}(T-s)^{k-\gamma_{2}-1} \hat{\Upsilon}\left(s, u_{0}(s)\right) d s \\
& \quad+C_{1} \Lambda_{1}+C_{2} \Lambda_{2}=\delta_{1},
\end{aligned}
$$

and

$$
\begin{aligned}
& \frac{\mu_{2}(\lambda-1)}{\lambda \Gamma\left(k-\theta+q_{1}\right)} \int_{0}^{T}(T-s)^{k-\theta+q_{1}-1} u_{0}(s) d s \\
& +\frac{\left(1-\mu_{2}\right)(\lambda-1)}{\lambda \Gamma\left(k-\theta+q_{2}\right)} \int_{0}^{T}(T-s)^{k-\theta+q_{2}-1} u_{0}(s) d s \\
& +\frac{\mu_{2}}{\lambda \Gamma\left(k+q_{1}\right)} \int_{0}^{T}(T-s)^{k+q_{1}-1} \hat{\Upsilon}\left(s, u_{0}(s)\right) d s \\
& +\frac{\left(1-\mu_{2}\right)}{\lambda \Gamma\left(k+q_{2}\right)} \int_{0}^{T}(T-s)^{k+q_{2}-1} \hat{\Upsilon}\left(s, u_{0}(s)\right) d s \\
& \quad+C_{1} \Lambda_{3}+C_{2} \Lambda_{4}=\delta_{2},
\end{aligned}
$$


which leads to

$$
\begin{aligned}
C_{1}= & \frac{\mu_{1} \Lambda_{4}(\lambda-1)}{\lambda} \mathcal{I}^{k-\theta-\gamma_{1}} u_{0}(T)-\frac{\Lambda_{2} \mu_{2}(\lambda-1)}{\lambda} \mathcal{I}^{k-\theta+q_{1}} u_{0}(T) \\
& +\frac{\Lambda_{4}\left(1-\mu_{1}\right)(\lambda-1)}{\lambda} \mathcal{I}^{k-\theta-\gamma_{2}} u_{0}(T)-\frac{\Lambda_{2}\left(1-\mu_{2}\right)(\lambda-1)}{\lambda} \mathcal{I}^{k-\theta+q_{2}} u_{0}(T) \\
& +\frac{\Lambda_{4} \mu_{1}}{\lambda} \mathcal{I}^{k-\gamma_{1}} \hat{\Upsilon}\left(T, u_{0}(T)\right)-\frac{\Lambda_{2} \mu_{2}}{\lambda} \mathcal{I}^{k+q_{1}} \hat{\Upsilon}\left(T, u_{0}(T)\right)+\Lambda_{2} \delta_{2}-\Lambda_{4} \delta_{1} \\
& +\frac{\Lambda_{4}\left(1-\mu_{1}\right)}{\lambda} \mathcal{I}^{k-\gamma_{2}} \hat{\Upsilon}\left(T, u_{0}(T)\right)-\frac{\Lambda_{2}\left(1-\mu_{2}\right)}{\lambda} \mathcal{I}^{k+q_{2}} \hat{\Upsilon}\left(T, u_{0}(T)\right)
\end{aligned}
$$

and

$$
\begin{aligned}
C_{2}= & \frac{\mu_{1} \Lambda_{3}(\lambda-1)}{\lambda} \mathcal{I}^{k-\theta-\gamma_{1}} u_{0}(T)-\frac{\Lambda_{1} \mu_{2}(\lambda-1)}{\lambda} \mathcal{I}^{k-\theta+q_{1}} u_{0}(T) \\
& +\frac{\Lambda_{3}\left(1-\mu_{1}\right)(\lambda-1)}{\lambda} \mathcal{I}^{k-\theta-\gamma_{2}} u_{0}(T)-\frac{\Lambda_{1}\left(1-\mu_{2}\right)(\lambda-1)}{\lambda} \mathcal{I}^{k-\theta+q_{2}} u_{0}(T) \\
& +\frac{\Lambda_{3} \mu_{1}}{\lambda} \mathcal{I}^{k-\gamma_{1}} \hat{\Upsilon}\left(T, u_{0}(T)\right)-\frac{\Lambda_{1} \mu_{2}}{\lambda} \mathcal{I}^{k-q_{1}} \hat{\Upsilon}\left(T, u_{0}(T)\right)+\Lambda_{1} \delta_{2}-\Lambda_{3} \delta_{1} \\
& +\frac{\Lambda_{3}\left(1-\mu_{1}\right)}{\lambda} \mathcal{I}^{k-\gamma_{2}} \hat{\Upsilon}\left(T, u_{0}(T)\right)-\frac{\Lambda_{1}\left(1-\mu_{2}\right)}{\lambda} \mathcal{I}^{k+q_{2}} \hat{\Upsilon}\left(T, u_{0}(T)\right) .
\end{aligned}
$$

By inserting the values of constants $C_{1}$ and $C_{2}$ in (8), we find that $u_{0}$ satisfies (5). Some calculations show that the converse part holds. This completes the proof.

Based on Lemma 7, define the operator $\mathcal{F}: \mathcal{C} \rightarrow \mathcal{C}$ by

$$
\begin{aligned}
\mathcal{F} u(t)= & \frac{\lambda-1}{\lambda \Gamma(k-\theta)} \int_{0}^{t}(t-s)^{k-\theta-1} u(s) d s+\frac{1}{\lambda \Gamma(k)} \int_{0}^{t}(t-s)^{k-1} \hat{\Upsilon}(t, u(t)) d s \\
& +\frac{t^{k-1}}{\Theta} \times\left[\frac{\mu_{1} \Lambda_{4}(\lambda-1)}{\lambda} \mathcal{I}^{k-\theta-\gamma_{1}} u(T)-\frac{\Lambda_{2} \mu_{2}(\lambda-1)}{\lambda} \mathcal{I}^{k-\theta+q_{1}} u(T)\right. \\
& +\frac{\Lambda_{2}\left(1-\mu_{1}\right)(\lambda-1)}{\lambda} \mathcal{I}^{k-\theta-\gamma_{2}} u(T)-\frac{\Lambda_{2}\left(1-\mu_{2}\right)(\lambda-1)}{\lambda} \mathcal{I}^{k-\theta+q_{2}} u(T) \\
& +\frac{\Lambda_{2} \mu_{1}}{\lambda} \mathcal{I}^{k-\gamma_{1}} \hat{\Upsilon}(T, u(T))-\frac{\Lambda_{2} \mu_{2}}{\lambda} \mathcal{I}^{k+q_{1}} \hat{\Upsilon}(T, u(T))+\Lambda_{2} \delta_{2}-\Lambda_{4} \delta_{1} \\
& \left.+\frac{\Lambda_{4}\left(1-\mu_{1}\right)}{\lambda} \mathcal{I}^{k-\gamma_{2}} \hat{\Upsilon}(T, u(T))-\frac{\Lambda_{2}\left(1-\mu_{2}\right)}{\lambda} \mathcal{I}^{k+q_{2}} \hat{\Upsilon}(T, u(T))\right] \\
& -\frac{t^{k-2}}{\Theta}\left[\frac{\mu_{1} \Lambda_{3}(\lambda-1)}{\lambda} \mathcal{I}^{k-\theta-\gamma_{1}} u(T)-\frac{\Lambda_{1} \mu_{2}(\lambda-1)}{\lambda} \mathcal{I}^{k-\theta+q_{1}} u(T)\right. \\
& +\frac{\Lambda_{3}\left(1-\mu_{1}\right)(\lambda-1)}{\lambda} \mathcal{I}^{k-\theta-\gamma_{2}} u(T)-\frac{\Lambda_{1}\left(1-\mu_{2}\right)(\lambda-1)}{\lambda} \mathcal{I}^{k-\theta+q_{2}} u(T) \\
& +\frac{\Lambda_{3} \mu_{1}}{\lambda} \mathcal{I}^{k-\gamma_{1}} \hat{\Upsilon}(T, u(T))-\frac{\Lambda_{1} \mu_{2}}{\lambda} \mathcal{I}^{k-q_{1}} \hat{\Upsilon}(T, u(T))+\Lambda_{1} \delta_{2}-\Lambda_{3} \delta_{1} \\
& \left.+\frac{\Lambda_{3}\left(1-\mu_{1}\right)}{\lambda} \mathcal{I}^{k-\gamma_{2}} \hat{\Upsilon}(T, u(T))-\frac{\Lambda_{1}\left(1-\mu_{2}\right)}{\lambda} \mathcal{I}^{k+q_{2}} \hat{\Upsilon}(T, u(T))\right]
\end{aligned}
$$


Note that boundary value problem (4) has solution $u_{0}$ if and only if $u_{0}$ is a fixed point of the operator $\mathcal{F} u$. To simplify calculations, we use the notations

$$
\begin{aligned}
\mathcal{W}_{1}= & \frac{(|\lambda-1|)\left(\Lambda_{4}+\Lambda_{3} T^{-1}\right)}{|\Theta|}\left(\mu_{1} \frac{T^{2 k-\theta-\gamma_{1}-1}}{\lambda \Gamma\left(k-\theta-\gamma_{1}+1\right)}+\frac{\left(1-\mu_{1}\right) T^{2 k-\theta-\gamma_{2}-1}}{\lambda \Gamma\left(k-\theta-\gamma_{2}+1\right)}\right) \\
& +\frac{(|\lambda-1|)\left(\Lambda_{2}+\Lambda_{1} T^{-1}\right)}{|\Theta|}\left(\mu_{2} \frac{T^{2 k-\theta+q_{1}-1}}{\lambda \Gamma\left(k-\theta+q_{1}+1\right)}+\frac{\left(1-\mu_{2}\right) T^{2 k-\theta+q_{2}-1}}{\lambda \Gamma\left(k-\theta+q_{2}+1\right)}\right) \\
& +\frac{(|\lambda-1|) T^{k-\theta}}{\lambda \Gamma(k-\theta+1)}
\end{aligned}
$$

and

$$
\begin{aligned}
\mathcal{W}_{2}= & \frac{T^{k}}{\lambda \Gamma(k+1)}+\frac{\Lambda_{4}+\Lambda_{3} T^{-1}}{|\Theta|}\left(\mu_{1} \frac{T^{2 k-\gamma_{1}-1}}{\lambda \Gamma\left(k-\gamma_{1}+1\right)}+\frac{\left(1-\mu_{1}\right) T^{2 k-\gamma_{2}-1}}{\lambda \Gamma\left(k-\gamma_{2}+1\right)}\right) \\
& +\frac{\Lambda_{2}+\Lambda_{1} T^{-1}}{|\Theta|}\left(\mu_{2} \frac{T^{2 k+q_{1}-1}}{\lambda \Gamma\left(k+q_{1}+1\right)}+\frac{\left(1-\mu_{2}\right) T^{2 k+q_{2}-1}}{\lambda \Gamma\left(k+q_{2}+1\right)}\right) .
\end{aligned}
$$

Theorem 8 Suppose that $\hat{\Upsilon}: J \times \mathbb{R} \rightarrow \mathbb{R}$ is a continuous map and there exists a constant $\mathcal{L}>0$ such that $\left|\hat{\Upsilon}(t, u)-\hat{\Upsilon}\left(t, u^{\prime}\right)\right| \leq \mathcal{L}\left|u-u^{\prime}\right|$ for all $t \in J$ and $u, u^{\prime} \in \mathbb{R}$. If $\mathcal{L} \mathcal{W}_{2}+\mathcal{W}_{1}<1$, then problem (4) has a unique solution, where $\mathcal{W}_{1}$ and $\mathcal{W}_{2}$ are defined by (10) and (11).

Proof Put $\sup _{t \in J}|\hat{\Upsilon}(t, 0)|=\mathcal{N}<\infty$ and choose

$$
\frac{|\Theta| \mathcal{N} \mathcal{R}+T^{k-1}\left(\left|\Lambda_{2} \delta_{2}\right|+\left|\Lambda_{4} \delta_{1}\right|\right)+T^{k-2}\left(\left|\Lambda_{1} \delta_{2}\right|+\left|\Lambda_{3} \delta_{1}\right|\right)}{|\Theta|\left(1-\mathcal{L} \mathcal{W}_{2}-\mathcal{W}_{1}\right)} \leq \mathcal{R}
$$

where $\Lambda_{i} i \in\{1,2,3,4\}$ are defined by (7). Set $\mathcal{B}_{\mathcal{R}}=\{u \in \mathcal{C}:\|u\| \leq \mathcal{R}\}$. We show that $\mathcal{F} \mathcal{B}_{\mathcal{R}} \subset \mathcal{B}_{\mathcal{R}}$. For each $u \in \mathcal{B}_{\mathcal{R}}$, we have

$$
\begin{aligned}
& |\mathcal{F} u(t)| \leq \frac{|\lambda-1|}{\lambda \Gamma(k-\theta)} \int_{0}^{t}(t-s)^{k-\theta-1}|u(s)| d s \\
& +\frac{1}{\lambda \Gamma(k)} \int_{0}^{t}(t-s)^{k-1}|\hat{\Upsilon}(t, u(t))-\hat{\Upsilon}(t, 0)|+|\hat{\Upsilon}(t, 0)| d s \\
& +\frac{T^{k-1}}{|\Theta|} \times\left[\frac{\mu_{1} \Lambda_{4}(|\lambda-1|)}{\lambda} \mathcal{I}^{k-\theta-\gamma_{1}}|u(T)|+\frac{\Lambda_{2} \mu_{2}(|\lambda-1|)}{\lambda} \mathcal{I}^{k-\theta+q_{1}}|u(T)|\right. \\
& +\frac{\Lambda_{4}\left(1-\mu_{1}\right)(|\lambda-1|)}{\lambda} \mathcal{I}^{k-\theta-\gamma_{2}}|u(T)|+\frac{\Lambda_{2}\left(1-\mu_{2}\right)(|\lambda-1|)}{\lambda} \mathcal{I}^{k-\theta+q_{2}}|u(T)| \\
& +\frac{\Lambda_{4} \mu_{1}}{\lambda} \mathcal{I}^{k-\gamma_{1}}(|\hat{\Upsilon}(T, u(T))-\hat{\Upsilon}(T, 0)|+|\hat{\Upsilon}(T, 0)|) \\
& +\frac{\Lambda_{2} \mu_{2}}{\lambda} \mathcal{I}^{k+q_{1}}(|\hat{\Upsilon}(T, u(T))-\hat{\Upsilon}(T, 0)|+|\hat{\Upsilon}(T, 0)|)+\Lambda_{2} \delta_{2}-\Lambda_{4} \delta_{1} \\
& +\frac{\Lambda_{4}\left(\left|1-\mu_{1}\right|\right)}{\lambda} \mathcal{I}^{k-\gamma_{2}}(|\hat{\Upsilon}(T, u(T))-\hat{\Upsilon}(T, 0)|+|\hat{\Upsilon}(T, 0)|) \\
& \left.+\frac{\Lambda_{2}\left(\left|1-\mu_{2}\right|\right)}{\lambda} \mathcal{I}^{k+q_{2}}(|\hat{\Upsilon}(T, u(T))-\hat{\Upsilon}(T, 0)|+|\hat{\Upsilon}(T, 0)|)\right] \\
& +\frac{T^{k-2}}{|\Theta|}\left[\frac{\mu_{1} \Lambda_{3}(\lambda-1)}{\lambda} \mathcal{I}^{k-\theta-\gamma_{1}}|u(T)|+\frac{\Lambda_{1} \mu_{2}(|\lambda-1|)}{\lambda} \mathcal{I}^{k-\theta+q_{1}}|u(T)|\right.
\end{aligned}
$$




$$
\begin{aligned}
& +\frac{\Lambda_{3}\left(1-\mu_{1}\right)(|\lambda-1|)}{\lambda} \mathcal{I}^{k-\theta-\gamma_{2}}|u(T)|+\frac{\Lambda_{1}\left(1-\mu_{2}\right)(|\lambda-1|)}{\lambda} \mathcal{I}^{k-\theta+q_{2}}|u(T)| \\
+ & \frac{\Lambda_{3} \mu_{1}}{\lambda} \mathcal{I}^{k-\gamma_{1}}(|\hat{\Upsilon}(T, u(T))-\hat{\Upsilon}(T, 0)|+|\hat{\Upsilon}(T, 0)|) \\
+ & \frac{\Lambda_{1} \mu_{2}}{\lambda} \mathcal{I}^{k-q_{1}}|\hat{\Upsilon}(T, u(T))-\hat{\Upsilon}(T, 0)|+|\hat{\Upsilon}(T, 0)|+\left|\Lambda_{1} \delta_{2}\right|+\left|\Lambda_{3} \delta_{1}\right| \\
& +\frac{\Lambda_{3}\left(1-\mu_{1}\right)}{\lambda} \mathcal{I}^{k-\gamma_{2}}(|\hat{\Upsilon}(T, u(T))-\hat{\Upsilon}(T, 0)|+|\hat{\Upsilon}(T, 0)|) \\
& \left.+\frac{\Lambda_{1}\left(1-\mu_{2}\right)}{\lambda} \mathcal{I}^{k+q_{2}}(|\hat{\Upsilon}(T, u(T))-\hat{\Upsilon}(T, 0)|+|\hat{\Upsilon}(T, 0)|)\right] \\
\leq & (\mathcal{L}\|u\|+\mathcal{N}) \mathcal{W}_{2}+\|u\| \mathcal{W}_{1} \\
& +\frac{1}{|\Theta|}\left[T^{k-1}\left(\left|\Lambda_{2} \delta_{2}\right|+\left|\Lambda_{4} \delta_{1}\right|\right)+T^{k-2}\left(\left|\Lambda_{1} \delta_{2}\right|+\left|\Lambda_{3} \delta_{1}\right|\right)\right] \\
= & \left(\mathcal{L} \mathcal{W}_{2}+\mathcal{W}_{1}\right) \mathcal{R}+\mathcal{N} \mathcal{W}_{2} \\
& +\frac{1}{|\Theta|}\left[T^{k-1}\left(\left|\Lambda_{2} \delta_{2}\right|+\left|\Lambda_{4} \delta_{1}\right|\right)+T^{k-2}\left(\left|\Lambda_{1} \delta_{2}\right|+\left|\Lambda_{3} \delta_{1}\right|\right)\right] \\
\leq & \mathcal{R} .
\end{aligned}
$$

Thus, $\|F u\| \leq \mathcal{R}$ and so $\mathcal{F} \mathcal{B}_{\mathcal{R}} \subset \mathcal{B}_{\mathcal{R}}$. Let $u, u^{\prime} \in \mathcal{C}$. For each $t \in J$, we have

$$
\begin{aligned}
& \left|\mathcal{F} u(t)-\mathcal{F} u^{\prime}(t)\right| \leq \frac{|\lambda-1|}{\lambda \Gamma(k-\theta)} \int_{0}^{t}(t-s)^{k-\theta-1}\left|u(s)-u^{\prime}(s)\right| d s \\
& +\frac{1}{\lambda \Gamma(k)} \int_{0}^{t}(t-s)^{k-1}\left|\hat{\Upsilon}(t, u(t))-\hat{\Upsilon}\left(t, u^{\prime}(s)\right)\right| d s \\
& +\frac{T^{k-1}}{|\Theta|} \times\left[\frac{\mu_{1} \Lambda_{4}(|\lambda|)}{\lambda} \mathcal{I}^{k-\theta-\gamma_{1}}\left|u(T)-u^{\prime}(T)\right|\right. \\
& +\frac{\Lambda_{2} \mu_{2}(|\lambda-1|)}{\lambda} \mathcal{I}^{k-\theta+q_{1}}\left|u(T)-u^{\prime}(T)\right| \\
& +\frac{\Lambda_{4}\left(1-\mu_{1}\right)(|\lambda-1|)}{\lambda} \mathcal{I}^{k-\theta-\gamma_{2}}\left|u(T)-u^{\prime}(T)\right| \\
& +\frac{\Lambda_{2}\left(1-\mu_{2}\right)(|\lambda-1|)}{\lambda} \mathcal{I}^{k-\theta+q_{2}}\left|u(T)-u^{\prime}(T)\right| \\
& +\frac{\Lambda_{4} \mu_{1}}{\lambda} \mathcal{I}^{k-\gamma_{1}}\left(\left|\hat{\Upsilon}(T, u(T))-\hat{\Upsilon}\left(T, u^{\prime}(T)\right)\right|\right) \\
& +\frac{\Lambda_{2} \mu_{2}}{\lambda} \mathcal{I}^{k+q_{1}}\left(\left|\hat{\Upsilon}(T, u(T))-\hat{\Upsilon}\left(T, u^{\prime}(T)\right)\right|\right) \\
& +\frac{\Lambda_{4}\left(\left|1-\mu_{1}\right|\right)}{\lambda} \times \mathcal{I}^{k-\gamma_{2}}\left(\left|\hat{\Upsilon}(T, u(T))-\hat{\Upsilon}\left(T, u^{\prime}(T)\right)\right|\right) \\
& \left.+\frac{\Lambda_{2}\left(\left|1-\mu_{2}\right|\right)}{\lambda} \mathcal{I}^{k+q_{2}}\left(\left|\hat{\Upsilon}(T, u(T))-\hat{\Upsilon}\left(T, u^{\prime}(T)\right)\right|\right)\right] \\
& +\frac{T^{k-2}}{|\Theta|}\left[\frac{\mu_{1} \Lambda_{3}(\lambda-1)}{\lambda} \mathcal{I}^{k-\theta-\gamma_{1}}\left|u(T)-u^{\prime}(T)\right|\right. \\
& +\frac{\Lambda_{1} \mu_{2}(|\lambda-1|)}{\lambda} \mathcal{I}^{k-\theta+q_{1}}\left|u(T)-u^{\prime}(T)\right|
\end{aligned}
$$




$$
\begin{aligned}
& +\frac{\Lambda_{3}\left(1-\mu_{1}\right)(|\lambda-1|)}{\lambda} \mathcal{I}^{k-\theta-\gamma_{2}}\left|u(T)-u^{\prime}(T)\right| \\
& +\frac{\Lambda_{1}\left(1-\mu_{2}\right)(|\lambda-1|)}{\lambda} \mathcal{I}^{k-\theta+q_{2}}\left|u(T)-u^{\prime}(T)\right| \\
& +\frac{\Lambda_{3} \mu_{1}}{\lambda} \mathcal{I}^{k-\gamma_{1}}\left(\left|\hat{\Upsilon}(T, u(T))-\hat{\Upsilon}\left(T, u^{\prime}(T)\right)\right|\right) \\
& +\frac{\Lambda_{1} \mu_{2}}{\lambda} \mathcal{I}^{k-q_{1}}\left|\hat{\Upsilon}(T, u(T))-\hat{\Upsilon}\left(T, u^{\prime}(T)\right)\right| \\
& +\frac{\Lambda_{3}\left(1-\mu_{1}\right)}{\lambda} \mathcal{I}^{k-\gamma_{2}}\left(\left|\hat{\Upsilon}(T, u(T))-\hat{\Upsilon}\left(T, u^{\prime}(T)\right)\right|\right) \\
& \left.+\frac{\Lambda_{1}\left(1-\mu_{2}\right)}{\lambda} \mathcal{I}^{k+q_{2}}\left(\left|\hat{\Upsilon}(T, u(T))-\hat{\Upsilon}\left(T, u^{\prime}(T)\right)\right|\right)\right] \\
& \leq\left(\mathcal{L}\left\|u-u^{\prime}\right\|\right) \mathcal{W}_{2}+\left\|u-u^{\prime}\right\| \mathcal{W}_{1} \\
& =\left(\mathcal{L} \mathcal{W}_{2}+\mathcal{W}_{1}\right)\left\|u-u^{\prime}\right\| .
\end{aligned}
$$

Hence, $\left\|\mathcal{F} u-\mathcal{F} u^{\prime}\right\| \leq\left(\mathcal{L} \mathcal{W}_{2}+\mathcal{W}_{1}\right)\left\|u-u^{\prime}\right\|$ and so $\mathcal{F}$ is a contraction. By using the principle of contraction, $\mathcal{F}$ has a unique fixed point which is the unique solution for problem (4).

Here, by using Krasnoselskii's fixed point theorem, we provide our next existence result.

Theorem 9 Suppose that $\hat{\Upsilon}: J \times \mathbb{R} \rightarrow \mathbb{R}$ is a continuous map and there exists a constant $\mathcal{L}>0$ such that $\left|\hat{\Upsilon}(t, u)-\hat{\Upsilon}\left(t, u^{\prime}\right)\right| \leq \mathcal{L}\left|u-u^{\prime}\right|$ for each $t \in J$ and $u, u^{\prime} \in \mathbb{R}$. If there is $\mathcal{V}(t) \in$ $C\left(J, \mathbb{R}^{+}\right)$such that $\hat{\Upsilon}(t, u) \leq \mathcal{V}(t)$ for all $(t, u) \in J \times \mathbb{R}$ and $\mathcal{W}_{1}<1$, then problem (4) has at least one solution. Here, $\mathcal{W}_{1}$ is given by $(10)$.

Proof Let $\|\mathcal{V}\|=\sup _{t \in J}|\mathcal{V}(t)|$. Consider the set $\mathcal{B}_{r}=\{u \in \mathcal{C}:\|u\| \leq r\}$, where

$$
\frac{|\Theta|\|v\| \mathcal{W}_{2}+T^{k-1}\left(\left|\Lambda_{2} \delta_{2}\right|+\left|\Lambda_{4} \delta_{1}\right|\right)+T^{\alpha-2}\left(\left|\Lambda_{1} \delta_{2}\right|+\left|\Lambda_{3} \delta_{1}\right|\right)}{|\Theta|\left(1-\mathcal{W}_{1}\right)} \leq r
$$

and $\Lambda_{1}, \Lambda_{2}, \Lambda_{3}, \Lambda_{4}$, and $\mathcal{W}_{1}$ are given by (7) and (10), respectively. For each $t \in J$, define the operators $\mathcal{F}_{1}$ and $\mathcal{F}_{2}$ on $\mathcal{B}_{r}$ by

$$
\begin{aligned}
\mathcal{F}_{1} u(t)= & \frac{\lambda-1}{\lambda \Gamma(k-\theta)} \int_{0}^{t}(t-s)^{k-\theta-1} u(s) d s \\
& +\frac{t^{k-1}}{\Theta} \times\left[\frac{\mu_{1} \Lambda_{4}(\lambda-1)}{\lambda} \mathcal{I}^{k-\theta-\gamma_{1}} u(T)-\frac{\Lambda_{2} \mu_{2}(\lambda-1)}{\lambda} \mathcal{I}^{k-\theta+q_{1}} u(T)\right. \\
& \left.+\frac{\Lambda_{4}\left(1-\mu_{1}\right)(\lambda-1)}{\lambda} \mathcal{I}^{k-\theta-\gamma_{2}} u(T)-\frac{\Lambda_{2}\left(1-\mu_{2}\right)(\lambda-1)}{\lambda} \mathcal{I}^{k-\theta+q_{2}} u(T)\right] \\
& -\frac{t^{k-2}}{\Theta}\left[\frac{\mu_{1} \Lambda_{3}(\lambda-1)}{\lambda} \mathcal{I}^{k-\theta-\gamma_{1}} u(T)-\frac{\Lambda_{1} \mu_{2}(\lambda-1)}{\lambda} \mathcal{I}^{k-\theta+q_{1}} u(T)\right. \\
& \left.+\frac{\Lambda_{3}\left(1-\mu_{1}\right)(\lambda-1)}{\lambda} \mathcal{I}^{k-\theta-\gamma_{2}} u(T)-\frac{\Lambda_{1}\left(1-\mu_{2}\right)(\lambda-1)}{\lambda} \mathcal{I}^{k-\theta+q_{2}} u(T)\right]
\end{aligned}
$$


and

$$
\begin{aligned}
\mathcal{F}_{2} u(t)= & \frac{1}{\lambda \Gamma(k)} \int_{0}^{t}(t-s)^{k-1} \hat{\Upsilon}(t, u(t)) d s+\frac{t^{k-1}}{\Theta} \\
& \times\left[\frac{\Lambda_{4} \mu_{1}}{\lambda} \mathcal{I}^{k-\gamma_{1}} \hat{\Upsilon}(T, u(T))-\frac{\Lambda_{2} \mu_{2}}{\lambda} \mathcal{I}^{k+q_{1}} \hat{\Upsilon}(T, u(T))+\Lambda_{2} \delta_{2}-\Lambda_{4} \delta_{1}\right. \\
& \left.+\frac{\Lambda_{4}\left(1-\mu_{1}\right)}{\lambda} \mathcal{I}^{k-\gamma_{2}} \hat{\Upsilon}(T, u(T))-\frac{\Lambda_{2}\left(1-\mu_{2}\right)}{\lambda} \mathcal{I}^{k+q_{2}} \hat{\Upsilon}(T, u(T))\right] \\
& -\frac{t^{k-2}}{\Theta}\left[+\frac{\Lambda_{3} \mu_{1}}{\lambda} \mathcal{I}^{k-\gamma_{1}} \hat{\Upsilon}(T, u(T))-\frac{\Lambda_{1} \mu_{2}}{\lambda} \mathcal{I}^{k-q_{1}} \hat{\Upsilon}(T, u(T))\right. \\
& +\frac{\Lambda_{3}\left(1-\mu_{1}\right)}{\lambda} \mathcal{I}^{k-\gamma_{2}} \hat{\Upsilon}(T, u(T)) \\
& \left.-\frac{\Lambda_{1}\left(1-\mu_{2}\right)}{\lambda} \mathcal{I}^{k+q_{2}} \hat{\Upsilon}(T, u(T))+\Lambda_{1} \delta_{2}-\Lambda_{3} \delta_{1}\right] .
\end{aligned}
$$

We show that $\mathcal{F}_{2} u+\mathcal{F}_{2} u^{\prime} \in \mathcal{B}_{r}$. Let $u, u^{\prime} \in \mathcal{B}_{r}$. Then we have

$$
\begin{aligned}
\mid \mathcal{F}_{1} u(t) & +F_{2} u^{\prime}(t) \mid \\
\leq & \|\mathcal{V}\|\left[\frac{T^{k}}{\lambda \Gamma(k+1)}+\frac{\Lambda_{4}+\Lambda_{3} T^{-1}}{|\Theta|}\left(\frac{\mu_{1} T^{2 k-\gamma_{1}-1}}{\lambda \Gamma\left(k-\gamma_{1}+1\right)}\right.\right. \\
& \left.\left.+\frac{\left(1-\mu_{1}\right) T^{2 k-\gamma_{2}-1}}{\lambda \Gamma\left(k-\gamma_{2}+1\right)}\right)+\frac{\Lambda_{2}+\Lambda_{1} T^{-1}}{|\Theta|}\left(\frac{\mu_{2} T^{2 k+q_{1}-1}}{\lambda \Gamma\left(k+q_{1}+1\right)}+\frac{\left(1-\mu_{2}\right) T^{2 k+q_{2}-1}}{\lambda \Gamma\left(k+q_{2}+1\right)}\right)\right] \\
& +\|u\|\left[\frac{(|\lambda-1|) T^{\alpha-\theta}}{\lambda \Gamma(\alpha-\theta+1)}+\frac{(|\lambda-1|)\left(\Lambda_{4}+\Lambda_{3} T^{-1}\right)}{|\Theta|}\left(\frac{\mu_{1} T^{2 k-\theta-\gamma_{1}-1}}{\lambda \Gamma\left(k-\theta-\gamma_{1}+1\right)}\right.\right. \\
& \left.+\frac{\left(1-\mu_{1}\right) T^{2 k-\theta-\gamma_{2}-1}}{\lambda \Gamma\left(k-\theta-\gamma_{2}+1\right)}\right)+\frac{(|\lambda-1|)\left(\Lambda_{2}+\Lambda_{1} T^{-1}\right)}{|\Theta|}\left(\frac{\mu_{2} T^{2 k-\theta+q_{1}-1}}{\lambda \Gamma\left(k-\theta+q_{1}+1\right)}\right. \\
& \left.\left.+\frac{\left(1-\mu_{2}\right) T^{2 k-\theta+q_{2}-1}}{\lambda \Gamma\left(k-\theta+q_{2}+1\right)}\right)\right]+\frac{1}{|\Theta|}\left[T^{k-1}\left(\left|\Lambda_{2} \delta_{2}\right|+\left|\Lambda_{4} \delta_{1}\right|\right)+T^{k-2}\left(\left|\Lambda_{1} \delta_{2}\right|+\left|\Lambda_{3} \delta_{1}\right|\right)\right] \\
\leq & \left(\|\mathcal{V}\| \mathcal{W}_{2}+r \mathcal{W}_{1}\right)+\frac{1}{|\Theta|}\left[T^{k-1}\left(\left|\Lambda_{2} \delta_{2}\right|+\left|\Lambda_{4} \delta_{1}\right|\right) T^{k-2}\left(\left|\Lambda_{1} \delta_{2}\right|+\left|\Lambda_{3} \delta_{1}\right|\right)\right] \leq r,
\end{aligned}
$$

and so $\mathcal{F}_{1} u+\mathcal{F}_{2} u^{\prime} \in \mathcal{B}_{r}$. Now, we prove $\mathcal{F}_{1}$ is a contraction. For every $u, u^{\prime} \in \mathcal{B}_{r}$, we have

$$
\begin{aligned}
\left|\mathcal{F}_{1} u(t)-\mathcal{F}_{1} u^{\prime}(t)\right| & \\
\leq & \frac{|\lambda-1|}{\lambda \Gamma(k-\theta)} \int_{0}^{t}(t-s)^{k-\theta-1}\left|u(s)-u^{\prime}(s)\right| d s \\
& +\frac{T^{k-1}}{|\Theta|} \times\left[\frac{\mu_{1} \Lambda_{4}(|\lambda-1|)}{\lambda} \mathcal{I}^{k-\theta-\gamma_{1}}\left|u(T)-u^{\prime}(T)\right|\right. \\
& +\frac{\Lambda_{2} \mu_{2}(|\lambda-1|)}{\lambda} \mathcal{I}^{k-\theta+q_{1}}\left|u(T)-u^{\prime}(T)\right|+\frac{\Lambda_{4}\left(1-\mu_{1}\right)(|\lambda-1|)}{\lambda} \\
& \left.\times \mathcal{I}^{k-\theta-\gamma_{2}}\left|u(T)-u^{\prime}(T)\right|+\frac{\Lambda_{2}\left(1-\mu_{2}\right)(|\lambda-1|)}{\lambda} \mathcal{I}^{k-\theta+q_{2}}\left|u(T)-u^{\prime}(T)\right|\right] \\
& +\frac{t^{k-2}}{|\Theta|}\left[\frac{\mu_{1} \Lambda_{3}(\lambda-1)}{\lambda} \mathcal{I}^{k-\theta-\gamma_{1}}\left|u(T)-u^{\prime}(T)\right|+\frac{\Lambda_{1} \mu_{2}(|\lambda-1|)}{\lambda} \mathcal{I}^{k-\theta+q_{1}}\left|u(T)-u^{\prime}(T)\right|\right.
\end{aligned}
$$




$$
\begin{aligned}
& +\frac{\Lambda_{3}\left(1-\mu_{1}\right)(|\lambda-1|)}{\nmid \lambda} \mathcal{I}^{k-\theta-\gamma_{2}}\left|u(T)-u^{\prime}(T)\right| \\
& \left.+\frac{\Lambda_{1}\left(1-\mu_{2}\right)(|\lambda-1|)}{\lambda} \mathcal{I}^{k-\theta+q_{2}}\left|u(T)-u^{\prime}(T)\right|\right] \leq \mathcal{W}_{1}\left\|u-u^{\prime}\right\| .
\end{aligned}
$$

Since $\mathcal{W}_{1}<1, \mathcal{F}_{1}$ is a contraction. Utilizing the continuity of the function $\hat{\Upsilon}$, we find that the operator $\mathcal{F}_{2}$ is continuous. If $u \in \mathcal{B}_{r}$, then

$$
\begin{aligned}
\left\|\mathcal{F}_{2} u\right\| \leq & \|\mathcal{V}\|\left(\frac{T^{k}}{\lambda \Gamma(k+1)}+\frac{\Lambda_{4}+\Lambda_{3} T^{-1}}{\left|\Lambda_{3} \Lambda_{2}-\Lambda_{1} \Lambda_{4}\right|}\left(\mu_{1} \frac{T^{2 k-\gamma_{1}-1}}{\lambda \Gamma\left(k-\gamma_{1}+1\right)}+\frac{\left(1-\mu_{1}\right) T^{2 k-\gamma_{2}-1}}{\lambda \Gamma\left(k-\gamma_{2}+1\right)}\right)\right. \\
& \left.+\frac{\Lambda_{2}+\Lambda_{1} T^{-1}}{\left|\Lambda_{3} \Lambda_{2}-\Lambda_{1} \Lambda_{4}\right|}\left(\mu_{2} \frac{T^{2 k+q_{1}-1}}{\lambda \Gamma\left(k+q_{1}+1\right)}+\frac{\left(1-\mu_{2}\right) T^{2 k+q_{2}-1}}{\lambda \Gamma\left(k+q_{2}+1\right)}\right)\right)=\mathcal{W}_{2}\|\mathcal{V}\| .
\end{aligned}
$$

This means that the operator $\mathcal{F}_{2}$ is uniformly bounded on $\mathcal{B}_{r}$. Now, we show that $\mathcal{F}_{2}$ is equicontinuous. Set $\sup _{t \in J, u \in \mathcal{B}_{r}}|\hat{\Upsilon}(t, u)|=\overline{\mathcal{M}}$. For each $t_{1}, t_{2}$ with $t_{2}>t_{1}$ and $u \in \mathcal{B}_{r}$, we have

$$
\begin{aligned}
& \left|\mathcal{F}_{2} u\left(t_{2}\right)-\mathcal{F}_{2} u\left(t_{1}\right)\right| \\
& =\mid \frac{1}{\lambda \Gamma(k)} \int_{0}^{t_{2}}\left(t_{2}-s\right)^{k-1} \hat{\Upsilon}(t, u(t)) d s-\frac{1}{\lambda \Gamma(k)} \int_{0}^{t_{1}}\left(t_{1}-s\right)^{k-1} \hat{\Upsilon}(t, u(t)) d s \\
& +\frac{t_{2}^{k-1}-t_{1}^{k-1}}{\Theta} \times\left[\frac{\Lambda_{4} \mu_{1}}{\lambda} \mathcal{I}^{k-\gamma_{1}} \hat{\Upsilon}(T, u(T))-\frac{\Lambda_{2} \mu_{2}}{\lambda} \mathcal{I}^{k+q_{1}} \hat{\Upsilon}(T, u(T))+\Lambda_{2} \delta_{2}-\Lambda_{4} \delta_{1}\right. \\
& \left.+\frac{\Lambda_{4}\left(1-\mu_{1}\right)}{\lambda} \mathcal{I}^{k-\gamma_{2}} \hat{\Upsilon}(T, u(T))-\frac{\Lambda_{2}\left(1-\mu_{2}\right)}{\lambda} \mathcal{I}^{k+q_{2}} \hat{\Upsilon}(T, u(T))\right] \\
& -\frac{t_{2}^{k-2}-t_{1}^{k-2}}{\Theta}\left[+\frac{\Lambda_{3} \mu_{1}}{\lambda} \mathcal{I}^{k-\gamma_{1}} \hat{\Upsilon}(T, u(T))-\frac{\Lambda_{1} \mu_{2}}{\lambda} \mathcal{I}^{k-q_{1}} \hat{\Upsilon}(T, u(T))\right. \\
& \left.+\frac{\Lambda_{3}\left(1-\mu_{1}\right)}{\lambda} \mathcal{I}^{k-\gamma_{2}} \hat{\Upsilon}(T, u(T))-\frac{\Lambda_{1}\left(1-\mu_{2}\right)}{\lambda} \mathcal{I}^{k+q_{2}} \hat{\Upsilon}(T, u(T))+\Lambda_{1} \delta_{2}-\Lambda_{3} \delta_{1}\right] \\
& \leq \frac{\overline{\mathcal{M}}\left(2\left(t_{2}-t_{1}\right)^{k}+\left|t_{2}^{k}-t_{1}^{k}\right|\right)}{\lambda \Gamma(k+1)} \\
& +\frac{t_{2}^{k-1}-t_{1}^{k-1}}{|\Theta|} \times\left[\frac{\overline{\mathcal{M}} \Lambda_{4} \mu_{1} T^{k-\gamma_{1}}}{\lambda \Gamma\left(k-\gamma_{1}+1\right)}+\frac{\overline{\mathcal{M}} \Lambda_{2} \mu_{2} T^{k+q_{1}}}{\lambda \Gamma\left(k+q_{1}+1\right)}+\left|\Lambda_{2} \delta_{2}\right|+\left|\Lambda_{4} \delta_{1}\right|\right. \\
& \left.+\frac{\overline{\mathcal{M}} \Lambda_{4}\left(\left|1-\mu_{1}\right|\right) T^{k-\gamma_{2}}}{\lambda \Gamma\left(k-\gamma_{2}+1\right)}+\frac{\overline{\mathcal{M}} \Lambda_{2}\left(\left|1-\mu_{2}\right|\right) T^{k+q_{2}}}{\lambda \Gamma\left(k+q_{2}+1\right)}\right] \\
& +\frac{t_{2}^{k-2}-t_{1}^{k-2}}{|\Theta|}\left[\frac{\overline{\mathcal{M}} \Lambda_{3} \mu_{1} T^{k-\gamma_{1}}}{\lambda \Gamma\left(k-\gamma_{1}+1\right)}+\frac{\overline{\mathcal{M}} \Lambda_{1} \mu_{2} T^{k-q_{1}}}{\lambda \Gamma\left(k-q_{1}+1\right)}\right. \\
& \left.+\frac{\overline{\mathcal{M}} \Lambda_{3}\left(\left|1-\mu_{1}\right|\right) T^{k-\gamma_{2}}}{\lambda \Gamma\left(k-\gamma_{2}\right)}+\frac{\overline{\mathcal{M}} \Lambda_{1}\left(\left|1-\mu_{2}\right|\right) T^{k+q_{2}}}{\lambda \Gamma\left(k+q_{2}+1\right)}+\left|\Lambda_{1} \delta_{2}\right|+\left|\Lambda_{3} \delta_{1}\right|\right] .
\end{aligned}
$$

The right-hand side of the above inequality tends to zero independently of $u$ as $t_{2}$ tends to $t_{1}$. Hence, $\mathcal{F}_{2}$ is equicontinuous, and so $\mathcal{F}_{2}$ is relatively compact on $\mathcal{B}_{r}$. Now, by using the Arzela-Ascoli theorem, $\mathcal{F}_{2}$ is compact on $\mathcal{B}_{r}$. Now, by using Theorem 3 , boundary value problem (4) has at least one solution.

Here, by applying the Leray-Schauder theorem, we give another existence result. 
Theorem 10 Suppose that $\hat{\Upsilon}: J \times \mathbb{R} \rightarrow \mathbb{R}$ is a continuous map and there are nondecreasing continuous function $\Psi:[0, \infty) \rightarrow(0, \infty)$ and $\Phi \in C\left(J, \mathbb{R}^{+}\right)$such that $|\hat{\Upsilon}(t, u)| \leq$ $\Phi(t) \Psi(\|u\|)$ for all $(t, u) \in J \times \mathbb{R}$. Assume that there exists a constant $\mathcal{Q}>0$ such that

$$
\frac{\mathcal{Q}|\Theta|}{\mathcal{Q}|\Theta| \mathcal{W}_{1}+\Psi(\mathcal{Q})\|\Phi\||\Theta| \mathcal{W}_{2}+T^{k-1}\left(\left|\Lambda_{2} \delta_{2}\right|+\left|\Lambda_{4} \delta_{1}\right|\right)+T^{k-2}\left(\left|\Lambda_{1} \delta_{2}\right|+\left|\Lambda_{3} \delta_{1}\right|\right)}>1,
$$

where $\mathcal{W}_{1}, \mathcal{W}_{2}$ are defined by (10) and (11), respectively. Then boundary value problem (4) has at least one solution.

Proof Consider the operator $\mathcal{F}$ defined by (9). We show that $\mathcal{F}$ maps bounded sets into bounded sets of $\mathcal{C}$. Let $\rho>0$ and $\mathcal{B}_{\rho}=\{u \in \mathcal{C}:\|u\| \leq \rho\}$ be a bounded ball in $\mathcal{C}$ and $t \in J$. Then we have

$$
\begin{aligned}
|\mathcal{F} u(t)| \leq & \sup _{t \in J} \mid \frac{\lambda-1}{\lambda-\theta} \int_{0}^{t}(t-s)^{k-\theta-1} u(s) d s+\frac{1}{\lambda \Gamma(k)} \int_{0}^{t}(t-s)^{k-1} \hat{\Upsilon}(t, u(t)) d s \\
& +\frac{t^{k-1}}{\Theta} \times\left[\frac{\mu_{1} \Lambda_{4}(\lambda-1)}{\lambda} \mathcal{I}^{k-\theta-\gamma_{1}} u(T)-\frac{\Lambda_{2} \mu_{2}(\lambda-1)}{\lambda} \mathcal{I}^{k-\theta+q_{1}} u(T)\right. \\
& +\frac{\Lambda_{4}\left(1-\mu_{1}\right)(\lambda-1)}{\lambda} \mathcal{I}^{k-\theta-\gamma_{2}} u(T)-\frac{\Lambda_{2}\left(1-\mu_{2}\right)(\lambda-1)}{\lambda} \mathcal{I}^{k-\theta+q_{2}} u(T) \\
& +\frac{\Lambda_{4} \mu_{1}}{\lambda} \mathcal{I}^{k-\gamma_{1}} \hat{\Upsilon}(T, u(T))-\frac{\Lambda_{2} \mu_{2}}{\lambda} \mathcal{I}^{k+q_{1}} \hat{\Upsilon}(T, u(T))+\Lambda_{2} \delta_{2}-\Lambda_{4} \delta_{1} \\
& \left.+\frac{\Lambda_{4}\left(1-\mu_{1}\right)}{\lambda} \mathcal{I}^{k-\gamma_{2}} \hat{\Upsilon}(T, u(T))-\frac{\Lambda_{2}\left(1-\mu_{2}\right)}{\lambda} \mathcal{I}^{k+q_{2}} \hat{\Upsilon}(T, u(T))\right] \\
& -\frac{t^{k-2}}{\Theta}\left[\frac{\mu_{1} \Lambda_{3}(\lambda-1)}{\lambda} \mathcal{I}^{k-\theta-\gamma_{1}} u(T)-\frac{\Lambda_{1} \mu_{2}(\lambda-1)}{\lambda} \mathcal{I}^{k-\theta+q_{1}} u(T)\right. \\
& +\frac{\Lambda_{3}\left(1-\mu_{1}\right)(\lambda-1)}{\lambda} \mathcal{I}^{k-\theta-\gamma_{2}} u(T)-\frac{\Lambda_{1}\left(1-\mu_{2}\right)(\lambda-1)}{\lambda} \mathcal{I}^{k-\theta+q_{2}} u(T) \\
& +\frac{\Lambda_{3} \mu_{1}}{\lambda} \mathcal{I}^{k-\gamma_{1}} \hat{\Upsilon}(T, u(T))-\frac{\Lambda_{1} \mu_{2}}{\lambda} \mathcal{I}^{k-q_{1}} \hat{\Upsilon}(T, u(T))+\Lambda_{1} \delta_{2}-\Lambda_{3} \delta_{1} \\
& \left.+\frac{\Lambda_{3}\left(1-\mu_{1}\right)}{\lambda} \mathcal{I}^{k-\gamma_{2}} \hat{\Upsilon}(T, u(T))-\frac{\Lambda_{1}\left(1-\mu_{2}\right)}{\lambda} \mathcal{I}^{k+q_{2}} \hat{\Upsilon}(T, u(T))\right] \\
\leq & \|\Phi\| \Psi(\|u\|) \mathcal{W}_{2}+\|u\| \mathcal{W}_{1} \\
& +\frac{1}{|\Theta|}\left[T^{k-1}\left(\left|\Lambda_{2} \delta_{2}\right|+\left|\Lambda_{4} \delta_{1}\right|\right)+T^{k-2}\left(\left|\Lambda_{1} \delta_{2}\right|+\left|\Lambda_{3} \delta_{1}\right|\right)\right],
\end{aligned}
$$

and consequently

$$
\begin{aligned}
\|\mathcal{F} u(t)\| \leq & \|\Phi\| \Psi(\|u\|) \mathcal{W}_{2}+\|u\| \mathcal{W}_{1} \\
& +\frac{1}{|\Theta|}\left[T^{k-1}\left(\left|\Lambda_{2} \delta_{2}\right|+\left|\Lambda_{4} \delta_{1}\right|\right)+T^{k-2}\left(\left|\Lambda_{1} \delta_{2}\right|+\left|\Lambda_{3} \delta_{1}\right|\right)\right] .
\end{aligned}
$$

Now, we prove that the operator $\mathcal{F}$ maps bounded sets into equicontinuous sets of $\mathcal{C}$. Assume that $t_{1}, t_{2} \in J$ with $t_{1}<t_{2}$ and $u \in \mathcal{B}_{\rho}$. Then we have

$$
\begin{aligned}
& \left|\mathcal{F} u\left(t_{2}\right)-\mathcal{F} u\left(t_{1}\right)\right| \\
& \quad \leq \frac{|\lambda-1|}{\lambda \Gamma(k-\theta)}\left(\left|\int_{0}^{t_{2}}\left(t_{2}-s\right)^{k-\theta-1} u(s) d s-\int_{0}^{t_{1}}\left(t_{1}-s\right)^{k-\theta-1} u(s) d s\right|\right)
\end{aligned}
$$




$$
\begin{aligned}
& +\frac{1}{\lambda \Gamma(k)}\left(\left|\int_{0}^{t_{2}}\left(t_{2}-s\right)^{k-1} \hat{\Upsilon}(t, u(s)) d s-\int_{0}^{t_{1}}\left(t_{1}-s\right)^{k-1} \hat{\Upsilon}(t, u(s)) d s\right|\right) \\
& +\frac{t_{2}^{k-1}-t_{1}^{k-1}}{|\Theta|} \times\left[\mid \frac{\mu_{1} \Lambda_{4}(\lambda-1)}{\lambda} \mathcal{I}^{k-\theta-\gamma_{1}} u(T)-\frac{\Lambda_{2} \mu_{2}(\lambda-1)}{\lambda} \mathcal{I}^{k-\theta+q_{1}} u(T)\right. \\
& +\frac{\Lambda_{4}\left(1-\mu_{1}\right)(\lambda-1)}{\lambda} \mathcal{I}^{k-\theta-\gamma_{2}} u(T)-\frac{\Lambda_{2}\left(1-\mu_{2}\right)(\lambda-1)}{\lambda} \mathcal{I}^{k-\theta+q_{2}} u(T) \\
& +\frac{\Lambda_{4} \mu_{1}}{\lambda} \mathcal{I}^{k-\gamma_{1}} \hat{\Upsilon}(T, u(T))-\frac{\Lambda_{2} \mu_{2}}{\lambda} \mathcal{I}^{k+q_{1}} \hat{\Upsilon}(T, u(T))+\Lambda_{2} \delta_{2}-\Lambda_{4} \delta_{1} \\
& \left.+\frac{\Lambda_{4}\left(1-\mu_{1}\right)}{\lambda} \mathcal{I}^{k-\gamma_{2}} \hat{\Upsilon}(T, u(T))-\frac{\Lambda_{2}\left(1-\mu_{2}\right)}{\lambda} \mathcal{I}^{k+q_{2}} \hat{\Upsilon}(T, u(T)) \mid\right] \\
& +\frac{t_{2}^{k-2}-t_{1}^{k-2}}{|\Theta|} \mid\left[\frac{\mu_{1} \Lambda_{3}(\lambda-1)}{\lambda} \mathcal{I}^{k-\theta-\gamma_{1}} u(T)-\frac{\Lambda_{1} \mu_{2}(\lambda-1)}{\lambda} \mathcal{I}^{k-\theta+q_{1}} u(T)\right. \\
& +\frac{\Lambda_{3}\left(1-\mu_{1}\right)(\lambda-1)}{\lambda} \mathcal{I}^{k-\theta-\gamma_{2}} u(T)-\frac{\Lambda_{1}\left(1-\mu_{2}\right)(\lambda-1)}{\lambda} \mathcal{I}^{k-\theta+q_{2}} u(T) \\
& +\frac{\Lambda_{3} \mu_{1}}{\lambda} \mathcal{I}^{k-\gamma_{1}} \hat{\Upsilon}(T, u(T))-\frac{\Lambda_{1} \mu_{2}}{\lambda} \mathcal{I}^{k-q_{1}} \hat{\Upsilon}(T, u(T))+\Lambda_{1} \delta_{2}-\Lambda_{3} \delta_{1} \\
& \left.+\frac{\Lambda_{3}\left(1-\mu_{1}\right)}{\lambda} \mathcal{I}^{k-\gamma_{2}} \hat{\Upsilon}(T, u(T))-\frac{\Lambda_{1}\left(1-\mu_{2}\right)}{\lambda} \mathcal{I}^{k+q_{2}} \hat{\Upsilon}(T, u(T))\right] \mid \\
& \leq \frac{|\lambda-1|}{\lambda \Gamma(k-\beta+1)}\left(\left(t_{2}^{k-\theta}-t_{1}^{k-\theta}\right)+2\left(t_{2}-t_{1}\right)^{k-\theta}\right) \\
& +\frac{1}{\lambda \Gamma(k+1)}\left(\left(t_{2}^{k}-t_{1}^{k}\right)+2\left(t_{2}-t_{1}\right)^{k}\right)+\frac{t_{2}^{k-1}-t_{1}^{k-1}}{|\Theta|} \\
& \times\left\{\| u \| \left[\frac{T^{k-\theta-\gamma_{1}}(|\lambda-1|)}{\lambda \Gamma\left(k-\theta-\gamma_{1}+1\right)}+\frac{T^{k-\theta+q_{1}}(|\lambda-1|) \Lambda_{2} \mu_{2}}{\lambda \Gamma\left(k-\theta+q_{1}+1\right)}\right.\right. \\
& \left.+\frac{\Lambda_{4}\left(1-\mu_{1}\right)(\lambda-1) T^{k-\theta-\gamma_{2}}}{\lambda \Gamma\left(k-\theta-\gamma_{2}+1\right)}+\frac{\Lambda_{2}\left(1-\mu_{2}\right)(|\lambda-1|) T^{k-\theta+q_{2}}}{\lambda \Gamma\left(k-\theta+q_{2}+1\right)}\right] \\
& +\overline{\mathcal{M}}\left[\Lambda_{4} \mu_{1} \frac{T^{k-\gamma_{1}}}{\lambda \Gamma\left(k-\gamma_{1}+1\right)}+\Lambda_{2} \mu_{2} \frac{T^{k+q_{1}}}{\lambda \Gamma\left(k+q_{1}+1\right)}\right. \\
& \left.\left.+\frac{\Lambda_{4}\left(1-\mu_{1}\right) T^{k-\gamma_{2}}}{\lambda \Gamma\left(k-\gamma_{2}+1\right)}+\frac{\Lambda_{2}\left(1-\mu_{2}\right) T^{k+q_{2}}}{\lambda \Gamma\left(k+q_{2}+1\right)}\right]+\left|\Lambda_{2} \delta_{2}\right|+\left|\Lambda_{4} \delta_{1}\right|\right\} \\
& +\frac{t_{2}^{k-2}-t_{1}^{k-2}}{|\Theta|}\left\{\| u \| \left[\mu_{1} \Lambda_{3} \frac{T^{k-\theta-\gamma_{1}}(|\lambda-1|)}{\lambda \Gamma\left(k-\theta-\gamma_{1}+1\right)}+\Lambda_{1} \mu_{2} \frac{T^{k-\theta+q_{1}}(|\lambda-1|)}{\lambda \Gamma\left(k-\theta+q_{1}+1\right)}\right.\right. \\
& \left.+\frac{\Lambda_{3}\left(1-\mu_{1}\right)(|\lambda-1|) T^{k-\theta-\gamma_{2}}}{\lambda \Gamma\left(k-\theta-\gamma_{2}+1\right)}+\frac{\Lambda_{1}\left(1-\mu_{2}\right)(|\lambda-1|) T^{k-\theta+q_{2}}}{\lambda \Gamma\left(k-\beta+q_{2}+1\right)}\right] \\
& +\overline{\mathcal{M}}\left[\Lambda_{3} \mu_{1} \frac{T^{k-\gamma_{1}}}{\lambda \Gamma\left(k-\gamma_{1}+1\right)}+\Lambda_{1} \mu_{2} \frac{T^{k+q_{1}}}{\lambda \Gamma\left(k+q_{1}+1\right)}\right. \\
& \left.\left.+\frac{\Lambda_{3}\left(1-\mu_{1}\right) T^{k-\gamma_{2}}}{\lambda \Gamma\left(k-\gamma_{2}+1\right)}+\frac{\Lambda_{1}\left(1-\mu_{2}\right) T^{k+q_{2}}}{\lambda \Gamma(k++1)}\right]+\left|\Lambda_{1} \delta_{2}\right|+\left|\Lambda_{3} \delta_{1}\right|\right\} \text {. }
\end{aligned}
$$

If $t_{2}-t_{1} \rightarrow 0$, then the right-hand side of the above inequality tends to zero independently of $x \in \mathcal{B}_{\rho}$. Thus, by using the Arzela-Ascoli theorem, the operator $\mathcal{F}: \mathcal{C} \rightarrow \mathcal{C}$ is completely continuous. The desired result is deduced from the Leray-Schauder theorem 4 once we prove the boundedness of the set of the solutions for the equation $u=\omega \mathcal{F} u$ for some 
$\omega \in(0,1)$. Let $u$ be a solution of the last equation. For each $t \in J$, we have

$$
\begin{aligned}
|u(t)| \leq & \|\Phi\| \Psi(\|u\|) \mathcal{W}_{2}+\|u\| \mathcal{W}_{1} \\
& +\frac{1}{|\Theta|}\left[T^{k-1}\left(\left|\Lambda_{2} \delta_{2}\right|+\left|\Lambda_{4} \delta_{1}\right|\right)+T^{k-2}\left(\left|\Lambda_{1} \delta_{2}\right|+\left|\Lambda_{3} \delta_{1}\right|\right)\right]
\end{aligned}
$$

and so $\frac{\|u\| \Theta \mid}{\|u\| \Theta\left|\mathcal{W}_{1}+\Psi(\|u\|)\|\Phi\| \Theta \Theta\right| \mathcal{W}_{2}+T^{k-1}\left(\left|\Lambda_{2} \delta_{2}\right|+\left|\Lambda_{4} \delta_{1}\right|\right)+T^{k-2}\left(\left|\Lambda_{1} \delta_{2}\right|+\left|\Lambda_{3} \delta_{1}\right|\right)}<1$. Choose the constant $\mathcal{Q}$ with $\|u\| \neq \mathcal{Q}$. Put $\mathcal{U}=\{x \in C:\|x\|<\mathcal{Q}\}$. One can check that the operator $\mathcal{F}: \overline{\mathcal{U}} \rightarrow \mathcal{C}$ is continuous and completely continuous. In view of the choice of $\mathcal{U}$, there is no $u \in \partial \mathcal{U}$ so that $u=\omega \mathcal{F} u$ for some $\omega \in(0,1)$. Now, by using the Leray-Schauder theorem, the operator $\mathcal{F}$ has a fixed point $u \in \overline{\mathcal{U}}$ which is a solution of boundary value problem (4).

\section{Stability analysis}

In this section, we study the Hyers-Ulam stability of the boundary value problem

$$
\left\{\begin{array}{l}
\lambda \mathcal{D}^{k}(u(t))+(1-\lambda) \mathcal{D}^{\theta}(u(t))=\hat{\Upsilon}(t, u(t)) \quad(t \in[0, T], k \in[2,3)) \\
u(0)=0, \quad \mathcal{D}^{\gamma_{1}} u(T)=\delta_{1}, \quad \mathcal{I}^{q_{1}} u(T)=\delta_{2}
\end{array}\right.
$$

which is a special case of problem (4) when we take $\mu_{1}=\mu_{2}=1$.

Definition 11 Problem (12) is called Hyers-Ulam stable whenever there exists a real constant $\ell>0$ such that, for each $\varepsilon>0$ and $u(t) \in C_{\mathbb{R}}([0, T])$ satisfying

$$
\left|\lambda \mathcal{D}^{k} u(t)+(1-\lambda) \mathcal{D}^{\theta}(u(t))-\hat{\Upsilon}(t, u(t))\right|<\varepsilon,
$$

there is a solution $v(t) \in C_{\mathbb{R}}([0, T])$ of problem (12) such that $|u(t)-v(t)| \leq \ell \varepsilon$ for all $t \in$ $[0, T]$.

Theorem 12 Suppose that $\hat{\Upsilon}: J \times \mathbb{R} \rightarrow \mathbb{R}$ is a continuous map and there exists a constant $\mathcal{L}>0$ such that $\left|\hat{\Upsilon}(t, u)-\hat{\Upsilon}\left(t, u^{\prime}\right)\right| \leq \mathcal{L}\left|u-u^{\prime}\right|$ for all $t \in J$ and $u, u^{\prime} \in \mathbb{R}$. Then boundary value problem (12) is Hyers-Ulam stable.

Proof Let $\varepsilon>0$ and $u(t) \in C_{\mathbb{R}}([0, T])$ be such that

$$
\left|\lambda \mathcal{D}^{k} u(t)+(1-\lambda) \mathcal{D}^{\theta}(u(t))-\hat{\Upsilon}(t, u(t))\right|<\varepsilon .
$$

Choose a function $g$ satisfying $\lambda \mathcal{D}^{k} u(t)+(1-\lambda) \mathcal{D}^{\theta}(u(t))=\hat{\Upsilon}(t, u(t))+g(t)$ and $|g(t)| \leq \varepsilon$ for all $t$. Then we have

$$
\begin{aligned}
u(t)= & \frac{\lambda-1}{\lambda} \mathcal{I}^{k-\theta} u(t)+\frac{1}{\lambda} \mathcal{I}^{k} \hat{\Upsilon}(t, u(t))+\frac{1}{\lambda} \mathcal{I}^{k} g(t) \\
& +\frac{t^{k-1}}{\Theta} \times\left[\frac{\Lambda_{4}(\lambda-1)}{\lambda} \mathcal{I}^{k-\theta-\gamma_{1}} u(T)-\frac{\Lambda_{2}(\lambda-1)}{\lambda} \mathcal{I}^{k-\theta+q_{1}} u(T)\right. \\
& +\frac{\Lambda_{4}}{\lambda} \mathcal{I}^{k-\gamma_{1}} \hat{\Upsilon}(T, u(T))-\frac{\Lambda_{2}}{\lambda} \mathcal{I}^{k+q_{1}} \hat{\Upsilon}(T, u(T))
\end{aligned}
$$




$$
\begin{aligned}
& \left.+\frac{\Lambda_{4}}{\lambda} \mathcal{I}^{k-\gamma_{1}} g(T)-\frac{\Lambda_{2}}{\lambda} \mathcal{I}^{k+q_{1}} g(T)+\Lambda_{2} \delta_{2}-\Lambda_{4} \delta_{1}\right] \\
& -\frac{t^{k-2}}{\Theta}\left[\frac{\Lambda_{3}(\lambda-1)}{\lambda} \mathcal{I}^{k-\theta-\gamma_{1}} u(T)-\frac{\Lambda_{1}(\lambda-1)}{\lambda} \mathcal{I}^{k-\theta+q_{1}} u(T)\right. \\
& +\frac{\Lambda_{3}}{\lambda} \mathcal{I}^{k-\gamma_{1}} \hat{\Upsilon}(T, u(T))-\frac{\Lambda_{1}}{\lambda} \mathcal{I}^{k+q_{1}} \hat{\Upsilon}(T, u(T)) \\
& \left.+\frac{\Lambda_{4}}{\lambda} \mathcal{I}^{k-\gamma_{1}} g(T)-\frac{\Lambda_{1}}{\lambda} \mathcal{I}^{k+q_{1}} g(T)+\Lambda_{1} \delta_{2}-\Lambda_{3} \delta_{1}\right]
\end{aligned}
$$

Let $v \in C_{\mathbb{R}}([0, T])$ be the unique solution of (12). Then $v$ is given by

$$
\begin{aligned}
v(t)= & \frac{\lambda-1}{\lambda} \mathcal{I}^{k-\theta} v(t)+\frac{1}{\lambda} \mathcal{I}^{k} \hat{\Upsilon}(t, v(t)) \\
& +\frac{t^{k-1}}{\Theta} \times\left[\frac{\Lambda_{4}(\lambda-1)}{\lambda} \mathcal{I}^{k-\theta-\gamma_{1}} v(T)-\frac{\Lambda_{2}(\lambda-1)}{\lambda} \mathcal{I}^{k-\theta+q_{1}} v(T)\right. \\
& \left.+\frac{\Lambda_{4}}{\lambda} \mathcal{I}^{k-\gamma_{1}} \hat{\Upsilon}(T, v(T))-\frac{\Lambda_{2}}{\lambda} \mathcal{I}^{k+q_{1}} \hat{\Upsilon}(T, v(T))+\Lambda_{2} \delta_{2}-\Lambda_{4} \delta_{1}\right] \\
& -\frac{t^{k-2}}{\Theta}\left[\frac{\Lambda_{3}(\lambda-1)}{\lambda} \mathcal{I}^{k-\theta-\gamma_{1}} v(T)-\frac{\Lambda_{1}(\lambda-1)}{\lambda} \mathcal{I}^{k-\theta+q_{1}} v(T)\right. \\
& \left.+\frac{\Lambda_{3}}{\lambda} \mathcal{I}^{k-\gamma_{1}} \hat{\Upsilon}(T, v(T))-\frac{\Lambda_{1}}{\lambda} \mathcal{I}^{k+q_{1}} \hat{\Upsilon}(T, v(T))+\Lambda_{1} \delta_{2}-\Lambda_{3} \delta_{1}\right] .
\end{aligned}
$$

Hence,

$$
\begin{aligned}
\mid u(t) & -v(t) \mid \\
\leq & \frac{|\lambda-1|}{\lambda} \mathcal{I}^{k-\theta}|u(t)-v(t)|+\frac{1}{\lambda} \mathcal{I}^{k}|\hat{\Upsilon}(t, u(t))-\hat{\Upsilon}(t, v(t))| \\
& +\frac{T^{k-1}}{|\Theta|} \times\left[\frac{\Lambda_{4}(\lambda-1)}{\lambda} \mathcal{I}^{k-\theta-\gamma_{1}}|u(T)-v(T)|+\frac{\Lambda_{2}(\lambda-1)}{\lambda} \mathcal{I}^{k-\theta+q_{1}}|u(T)-v(T)|\right. \\
& \left.+\frac{\Lambda_{4}}{\lambda} \mathcal{I}^{k-\gamma_{1}}|\hat{\Upsilon}(T, u(T))-\hat{\Upsilon}(T, v(T))|+\frac{\Lambda_{2}}{\lambda} \mathcal{I}^{k+q_{1}}|\hat{\Upsilon}(T, u(T))-\hat{\Upsilon}(T, v(T))|\right] \\
& +\frac{T^{k-2}}{|\Theta|}\left[\frac{\Lambda_{3}(\lambda-1)}{\lambda} \mathcal{I}^{k-\theta-\gamma_{1}}|u(T)-v(T)|+\frac{\Lambda_{1}(\lambda-1)}{\lambda} \mathcal{I}^{k-\theta+q_{1}}|u(T)-v(T)|\right. \\
& \left.+\frac{\Lambda_{3}}{\lambda} \mathcal{I}^{k-\gamma_{1}}|\hat{\Upsilon}(T, u(T))-\hat{\Upsilon}(T, v(T))|+\frac{\Lambda_{1}}{\lambda} \mathcal{I}^{k+q_{1}}|\hat{\Upsilon}(T, u(T))-\hat{\Upsilon}(T, v(T))|\right] \\
& +\frac{1}{\lambda} \mathcal{I}^{k}|g(t)|+\frac{T^{k-1}}{\Theta}\left(\frac{\Lambda_{4}}{\lambda} \mathcal{I}^{k-\gamma_{1}}|g(T)|+\frac{\Lambda_{2}}{\lambda} \mathcal{I}^{k+q_{1}}|g(T)|\right) \\
& +\frac{T^{k-2}}{\Theta}\left(\frac{\Lambda_{3}}{\lambda} \mathcal{I}^{k-\gamma_{1}}|g(T)|+\frac{\Lambda_{1}}{\lambda} \mathcal{I}^{k+q_{1}}|g(T)|\right) \\
\leq & \frac{1}{\lambda} \int_{0}^{t}\left[|\lambda-1| \frac{(t-s)^{k-\theta-1}}{\Gamma(k-\theta)}+\mathcal{L} \frac{(t-s)^{k-1}}{\Gamma(k)}\right]|u(s)-v(s)| d s+\overline{\mathcal{G}}(\varepsilon) \varepsilon \\
& +\left[\frac{T^{k}}{\lambda \Gamma(k+1)}+\frac{T^{2 k-\gamma_{1}-1} \Lambda_{4}}{\lambda \Theta \Gamma\left(k-\gamma_{1}+1\right)}+\frac{T^{k+q_{1}-1} \Lambda_{2}}{\lambda \Theta \Gamma\left(k+q_{1}+1\right)}\right. \\
& \left.+\frac{T^{2 k-\gamma_{1}-2} \Lambda_{3}}{\lambda \Theta \Gamma\left(k-\gamma_{1}+1\right)}+\frac{T^{2 k+q_{1}-2} \Lambda_{1}}{\lambda \Theta \Gamma\left(k+q_{1}+1\right)}\right] \varepsilon,
\end{aligned}
$$


where

$$
\begin{aligned}
\int_{0}^{T}\{ & \frac{\Lambda_{4}(\lambda-1) T^{k-1}}{\lambda|\Theta|} \frac{(t-s)^{k-\theta-\gamma_{1}-1}}{\Gamma\left(k-\theta-\gamma_{1}\right)}+\frac{\Lambda_{2}(\lambda-1) T^{k-1}}{\lambda|\Theta|}+\frac{(t-s)^{k-\theta+q_{1}-1}}{\Gamma\left(k-\theta-q_{1}\right)} \\
& +\frac{\Lambda_{4} T^{k-1}}{\lambda|\Theta|} \frac{(t-s)^{k-\gamma_{1}-1}}{\Gamma\left(k-\gamma_{1}\right)} \mathcal{L}+\frac{\Lambda_{2} T^{k-1}}{\lambda|\Theta|} \frac{(t-s)^{k+q_{1}-1}}{\Gamma\left(k+q_{1}\right)} \mathcal{L} \\
& +\frac{\Lambda_{3}(\lambda-1) T^{k-2}}{\lambda|\Theta|} \frac{(t-s)^{k-\theta-\gamma_{1}-1}}{\Gamma\left(k-\theta-\gamma_{1}\right)}+\frac{\Lambda_{1}(\lambda-1) T^{k-2}}{\lambda|\Theta|}+\frac{(t-s)^{k-\theta+q_{1}-1}}{\Gamma\left(k-\theta-q_{1}\right)} \\
& \left.+\frac{\Lambda_{3} T^{k-2}}{\lambda|\Theta|} \frac{(t-s)^{k-\gamma_{1}-1}}{\Gamma\left(k-\gamma_{1}\right)} \mathcal{L}+\frac{\Lambda_{1} T^{k-2}}{\lambda|\Theta|} \frac{(t-s)^{k+q_{1}-1}}{\Gamma\left(k+q_{1}\right)} \mathcal{L}\right\}|u(s)-v(t)| d s \\
\leq & \overline{\mathcal{G}}(\varepsilon) \varepsilon
\end{aligned}
$$

and $\overline{\mathcal{G}}(\varepsilon)$ is a constant dependent on $\varepsilon$. Let $g(t, s)=\frac{|\lambda-1|}{\lambda} \frac{1}{\Gamma(k-\theta)}+\mathcal{L} \frac{(t-s)^{\theta}}{\lambda \Gamma(k)}$ and

$$
\begin{aligned}
\Delta= & \overline{\mathcal{G}}(\varepsilon)+\left[\frac{T^{k}}{\lambda \Gamma(k+1)}+\frac{T^{2 k-\gamma_{1}-1} \Lambda_{4}}{\lambda \Theta \Gamma\left(k-\gamma_{1}+1\right)}+\frac{T^{2 k+q_{1}-1} \Lambda_{2}}{\lambda \Theta \Gamma\left(k+q_{1}+1\right)}\right. \\
& \left.+\frac{T^{2 k-\gamma_{1}-2} \Lambda_{3}}{\lambda \Theta \Gamma\left(k-\gamma_{1}+1\right)}+\frac{T^{2 k+q q_{1}-2} \Lambda_{1}}{\lambda \Theta \Gamma\left(k+q_{1}+1\right)}\right] .
\end{aligned}
$$

Then $|u(t)-v(t)| \leq \Delta \varepsilon+\int_{0}^{t} g(t, s)(t-s)^{\alpha-\theta-1}|u(s)-v(t)| d s$. Note that

$$
g(t, s) \leq \frac{|\lambda-1|}{\lambda} \frac{1}{\Gamma(k-\theta)}+\mathcal{L} \frac{T^{\theta}}{\lambda \Gamma(k)}=M
$$

In view of Lemma 5, we get

$$
\begin{aligned}
|u(t)-v(t)| & \leq \Delta \varepsilon+\Delta \varepsilon \int_{0}^{t} \sum_{n=1}^{\infty} \frac{(g(t, s) \Gamma(k-\theta))^{n}}{\Gamma(n(k-\theta))}(t-s)^{n(k-\theta)-1} d s \\
& \leq \Delta \varepsilon+\Delta \varepsilon \int_{0}^{t} \sum_{n=1}^{\infty} \frac{(M \Gamma(k-\theta))^{n}}{\Gamma(n(k-\theta))}(t-s)^{n(k-\theta)-1} d s \\
& \leq \Delta \varepsilon+\Delta \varepsilon \sum_{n=1}^{\infty} \frac{(M \Gamma(k-\theta))^{n}}{\Gamma(n(k-\theta)+1)} T^{n(k-\theta)} \\
& \leq \Delta \varepsilon E_{k-\theta}\left(M T^{(k-\theta)} \Gamma(k-\theta)\right) .
\end{aligned}
$$

Put $c=E_{k-\theta}\left(M T^{(k-\theta)} \Gamma(k-\theta)\right)$. Note that the inequality $|u(t)-v(t)|<c \varepsilon$ holds. Thus, boundary value problem (12) is Hyers-Ulam stable.

\section{Examples}

Now, we provide two examples to illustrate our main results. 
Example 1 Consider the boundary value problem

$$
\left\{\begin{array}{l}
\frac{47}{54} \mathcal{D}^{5 / 2} u(t)+\frac{7}{54} \mathcal{D}^{2.03} u(t)=t^{2} \cos (u(t)), \quad t \in\left[0, \frac{1}{4}\right] \\
u(0)=0 \\
\mu_{1} \mathcal{D}^{7 / 15} u\left(\frac{1}{4}\right)+\left(1-\mu_{1}\right) \mathcal{D}^{1 / 8} u\left(\frac{1}{4}\right)=\frac{1}{16} \\
\mu_{2} \mathcal{I}^{3 / 4} u\left(\frac{1}{4}\right)+\left(1-\mu_{2}\right) \mathcal{I}^{5 / 3} u\left(\frac{1}{4}\right)=\frac{5}{12}
\end{array}\right.
$$

Put $\lambda=47 / 54, k=5 / 2, \theta=2.1, \gamma_{1}=7 / 15, \gamma_{2}=1 / 8, q_{1}=3 / 4, q_{2}=5 / 3, \delta_{1}=1 / 16, \delta_{2}=5 / 12$, and $T=1 / 4$. Note that $0<\gamma_{1}, \gamma_{2}<0.47=k-\theta$ and

$$
\left|\hat{\Upsilon}(t, u(t))-\Upsilon\left(t, u^{\prime}(t)\right)\right| \leq\left(\frac{1}{4}\right)^{2}\left|u(t)-u^{\prime}(t)\right|
$$

with $\mathcal{L}=1 / 16$ and $|\hat{\Upsilon}(t, u(t))| \leq t^{2}=\mathcal{V}(t)$. If $\mu_{1}=1 / 3$ and $\mu_{2}=3 / 4$, then

$$
\begin{aligned}
& \Lambda_{1} \approx 0.2120, \quad \Lambda_{2} \approx 0.6825, \quad \Lambda_{3} \approx 0.0178, \quad \Lambda_{4} \approx 0.1084, \\
& \Theta \approx 0.0108, \quad \mathcal{W}_{1} \approx 0.6761, \quad \mathcal{W}_{2} \approx 0.0751 .
\end{aligned}
$$

Hence, $\mathcal{L} \mathcal{W}_{2}+\mathcal{W}_{1} \approx 0.6808<1$. Now, by using Theorem 8 , problem (13) has a unique solution. If $\mu_{1}=1$ and $\mu_{2}=1$, then $g(t, s)=\frac{|\lambda-1|}{\lambda} \frac{1}{\Gamma(k-\theta)}+\mathcal{L} \frac{(t-s)^{\theta}}{\lambda \Gamma(k)}$. Note that

$$
|g(t, s)| \leq \frac{|\lambda-1|}{\lambda} \frac{1}{\Gamma(k-\theta)}+\mathcal{L} \frac{T^{\theta}}{\lambda \Gamma(k)} \approx 0.0823=M .
$$

Now, by using Theorem 12, problem (2) is Hyers-Ulam stable.

Example 2 Consider the boundary value problem

$$
\left\{\begin{array}{l}
\frac{40}{45} \mathcal{D}^{13 / 5} u(t)+\frac{5}{45} \mathcal{D}^{2.01} u(t)=\frac{1}{t^{2}+5}\left(\frac{u^{2}(t)}{|u(t)|+1}+4\right), \quad t \in\left[0, \frac{1}{4}\right] \\
u(0)=0 \\
\frac{11}{33} \mathcal{D}^{7 / 88} u\left(\frac{1}{4}\right)+\frac{22}{33} \mathcal{D}^{3 / 100} u\left(\frac{1}{4}\right)=\frac{21}{156} \\
\frac{9}{13} \mathcal{I}^{5 / 4} u\left(\frac{1}{4}\right)+\frac{4}{13} \mathcal{I}^{23 / 33} u\left(\frac{1}{4}\right)=\frac{55}{122}
\end{array}\right.
$$

Put $\lambda=40 / 45, k=13 / 5, \theta=2.01, \gamma_{1}=7 / 88, \gamma_{2}=3 / 100, q_{1}=5 / 4, q_{2}=23 / 33, \delta_{1}=21 / 156$, $\delta_{2}=55 / 122$, and $T=\frac{1}{4}$. Note that $0<\gamma_{1}, \gamma_{2}<0.59=k-\theta$ and

$$
\hat{\Upsilon}(t, u(t))=\frac{1}{t^{2}+5}\left(\frac{u^{2}(t)}{|u(t)|+1}+4\right)
$$

Assume that $\mu_{1}=11 / 33$ and $\mu_{2}=9 / 13$. Then we have

$$
\begin{aligned}
& \Lambda_{1} \approx 0.1203, \quad \Lambda_{2} \approx 0.4667, \quad \Lambda_{3} \approx 0.0106, \quad \Lambda_{4} \approx 0.0663, \\
& \Theta \approx 0.0030, \quad \mathcal{W}_{1} \approx 0.6462, \quad \mathcal{W}_{2} \approx 0.0652,
\end{aligned}
$$


and $|\hat{\Upsilon}(t, u(t))|=\left|\frac{1}{t^{2}+5}\left(\frac{u^{2}(t)}{|u(t)|+1}+4\right)\right| \leq \frac{1}{t^{2}+5}(|x|+4)$. Put $\Phi(t)=\frac{1}{t^{2}+5}$ and $\Psi(|u|)=|x|+4$ and choose $\mathcal{Q}>379.5499$ such that

$$
\frac{\mathcal{Q}|\Theta|}{\mathcal{Q}|\Theta| \mathcal{W}_{1}+\Psi(\mathcal{Q})\|\Phi\||\Theta| \mathcal{W}_{2}+T^{k-1}\left(\left|\Lambda_{2} \delta_{2}\right|+\left|\Lambda_{4} \delta_{1}\right|\right)+T^{k-2}\left(\left|\Lambda_{1} \delta_{2}\right|+\left|\Lambda_{3} \delta_{1}\right|\right)}>1
$$

Now, by using Theorem 10, problem (14) has at least one solution.

\section{Conclusion}

As many researchers would like to investigate the stability notion of different boundary value problems, this can be a motivation for us to study the stability of complicated systems supplemented with general boundary conditions. Hence, our main goal in the present manuscript is to obtain some existence criteria of a new general boundary value problem including 2-term fractional differential equation which contains multi-order RiemannLiouville fractional derivatives and integrals. In the sequel, we check Hyers-Ulam stability of the proposed problem in the special case $\mu_{1}=1$ and $\mu_{2}=1$. Finally, we provide two illustrative examples to support our theoretical findings. This work can be an introduction for other researchers to study mentioned notions for numerous fractional multi-order modelings in the future.

\section{Acknowledgements}

The first and second authors were supported by University of Kasdi Merbah. The third and fourth authors were supported by Azarbaijan Shahid Madani University. The authors express their gratitude to dear unknown referees for their helpful suggestions which improved the final version of this paper.

Funding

Not applicable.

Availability of data and materials

Data sharing not applicable to this article as no datasets were generated or analyzed during the current study.

Ethics approval and consent to participate

Not applicable.

Competing interests

The authors declare that they have no competing interests.

Consent for publication

Not applicable.

Authors' contributions

The authors declare that the study was realized in collaboration with equal responsibility. All authors read and approved the final manuscript.

Authors' information

(Salim Ben Chikh: benchikh.salim@univ-ouargla.dz) (Abdelkader Amara: amara.abdelkader@univ-ouargla.dz) (Sina

Etemad: sina.etemad@gmail.com)

\section{Author details}

'Laboratory of Applied Mathematics, University of Kasdi Merbah, Ouargla 30000, Algeria. '2Department of Mathematics, Azarbaijan Shahid Madani University, Tabriz, Iran. ${ }^{3}$ Institute of Research and Development, Duy Tan University, Da Nang 550000, Vietnam. ${ }^{4}$ Faculty of Natural Sciences, Duy Tan University, Da Nang 550000, Vietnam. ${ }^{5}$ Department of Medical Research, China Medical University Hospital, China Medical University, Taichung, Taiwan.

\section{Publisher's Note}

Springer Nature remains neutral with regard to jurisdictional claims in published maps and institutional affiliations. 


\section{References}

1. Kilbas, A.A., Srivastava, H.M., Trujillo, J.J.: Theory and Applications of Fractional Differential Equations. Elsevier, Amsterdam (2006)

2. Lakshmikantham, V., Leela, S., Vasundhara, D.J.: Theory of Fractional Dynamic Systems. Cambridge Academic Publishers, London (2009)

3. Lakshmikantham, V., Vatsala, A.S.: Basic theory of fractional differential equations. Nonlinear Anal. 69, 2677-2682 (2008)

4. Miller, K.S., Ross, B.: An Introduction to the Fractional Calculus and Fractional Differential Equations. Wiley, New York (1993)

5. Rida, S.Z., Arafa, A.A.M., Gaber, Y.A.: Solution of the fractional epidemic model by L-ADM. J. Fract. Calc. Appl. 7(1), 189-195 (2016)

6. Baleanu, D., Etemad, S., Rezapour, S.: A hybrid Caputo fractional modeling for thermostat with hybrid boundary value conditions. Bound. Value Probl. 2020, 64 (2020). https://doi.org/10.1186/s13661-020-01361-0

7. Baleanu, D., Jajarmi, A., Mohammadi, H., Rezapour, S.: Analysis of the human liver model with Caputo-Fabrizio fractional derivative. Chaos Solitons Fractals 134, 7 (2020)

8. Baleanu, D., Aydogan, S.M., Mohammadi, H., Rezapour, S.: On modelling of epidemic childhood diseases with the Caputo-Fabrizio derivative by using the Laplace Adomian decomposition method. Alex. Eng. J. (2020). https://doi.org/10.1016/j.aej.2020.05.007

9. Baleanu, D., Rezapour, S., Mohammadi, H.: Some existence results on nonlinear fractional differential equations. Philos. Trans. R. Soc. A 371, 20120144 (2013). https://doi.org/10.1098/rsta.2012.0144

10. Etemad, S., Rezapour, S., Samei, M.E.: On a fractional Caputo-Hadamard inclusion problem with sum boundary value conditions by using approximate endpoint property. Math. Model. Appl. Sci. (2020). https://doi.org/10.1002/mma.6644

11. Tuan, N.H., Mohammadi, H., Rezapour, S.: A mathematical model for COVID-19 transmission by using the Caputo fractional derivative. Chaos Solitons Fractals 140, 110107 (2020). https://doi.org/10.1016/j.chaos.2020.110107

12. Almeida, R., Malinowska, A.B., Odzijewicz, T.: On systems of fractional differential equations with the $\psi$-Caputo derivative and their applications. Math. Methods Appl. Sci. (2019). https://doi.org/10.1002/mma.5678

13. Singh, H., Dhar, J., Bhatti, H.S., Chandok, S.: An epidemic model of childhood disease dynamics with maturation delay and latent period of infection. Model. Earth Syst. Environ. 2, 79 (2016)

14. Bao, N.T., Hoang, L.N., Van, A.V., Nguyen, H.T., Zhou, Y.: Existence and regularity of inverse problem for the nonlinear fractional Rayleigh-Stokes equations. Math. Methods Appl. Sci. (2019). https://doi.org/10.1002/mma.6162

15. Ntouyas, S.K., Tariboon, J.: Fractional boundary value problems with multiply orders of fractional derivatives and integrals. Electron. J. Differ. Equ. 2017, 100 (2017)

16. Baleanu, D., Etemad, S., Rezapour, S.: On a fractional hybrid integro-differential equation with mixed hybrid integral boundary value conditions by using three operators. Alex. Eng. J. (2020). https://doi.org/10.1016/j.aej.2020.04.053

17. Baleanu, D., Nazemi, Z., Rezapour, S.: Attractivity for a k-dimensional system of fractional functional differential equations and global attractivity for a k-dimensional system of nonlinear fractional differential equations. J. Inequal. Appl. 2014, 31 (2014). https://doi.org/10.1186/1029-242X-2014-31

18. Ghorbanian, R., Hedayati, V., Postolache, M., Rezapour, S.: On a fractional differential inclusion via a new integral boundary condition. J. Inequal. Appl. 2014,319 (2014). https://doi.org/10.1186/1029-242X-2014-319

19. Baleanu, D., Rezapour, S., Etemad, S., Alsaedi, A.: On a time-fractional partial integro-differential equation via three-point boundary value conditions. Math. Probl. Eng. 2015, Article ID 785738 (2015). https://doi.org/10.1155/2015/785738

20. Agarwal, R.P., Baleanu, D., Hedayati, V., Rezapour, S.: Two fractional derivative inclusion problems via integral boundary conditions. Appl. Math. Comput. 257, 205-212 (2015). https://doi.org/10.1016/j.amc.2014.10.082

21. Alsaedi, A., Baleanu, D., Etemad, S., Rezapour, S.: On coupled systems of time-fractional differential problems by using a new fractional derivative. J. Funct. Spaces 2016, Article ID 4626940 (2016). https://doi.org/10.1155/2016/4626940

22. Hedayati, V., Rezapour, S.: The existence of solution for a k-dimensional system of fractional differential inclusions with anti-periodic boundary value problems. Filomat 30(6), 1601-1613 (2016). https://doi.org/10.2298/FIL1606601H

23. Baleanu, D., Hedayati, V., Rezapour, S.: On two fractional differential inclusions. SpringerPlus 5, 882 (2016) https://doi.org/10.1186/s40064-016-2564-z

24. Aydogan, S.M., Nazemi, Z., Rezapour, S.: Positive solutions for a sum-type singular fractional integro-differential equation with m-point boundary conditions. UPB Sci. Bull., Ser. A, Appl. Math. Phys. 79(1), 89-98 (2017)

25. Rezapour, S., Samei, M.E.: On the existence of solutions for a multi-singular pointwise defined fractional q-integro-differential equation. Bound. Value Probl. 2020, 38 (2020). https://doi.org/10.1186/s13661-020-01342-3

26. Samei, M.E., Rezapour, S.: On a system of fractional $q$-differential inclusions via sum of two multi-term functions on a time scale. Bound. Value Probl. 2020, 135 (2020). https://doi.org/10.1186/s13661-020-01433-1

27. Bryan, G.H.: On the stability of a plane plate under thrusts in its own plane, with applications to the 'Buckling' of the sides of a ship. Proc. Lond. Math. Soc. 22, 54-67 (1890/91)

28. Greenhill, A.G.: Stability of orbits. Proc. Lond. Math. Soc. 22, 264-305 (1890/91)

29. Klein, F.: On the stability of a sleeping top. Bull. Am. Math. Soc. 3(4), 129-132 (1897)

30. Lockwood, E.H.: Atmospheric stability as affected by water vapor. Ph.D. thesis, Yale University, Ann Arbor, MI (1901)

31. Love, A.E.H.: Note on the stability of a thin elastic rod. Proc. Lond. Math. Soc. 24, 156-161 (1892/93)

32. Love, A.E.H.: On the stability of certain vortex motions. Proc. Lond. Math. Soc. 25, 18-42 (1893/94)

33. Rayleigh, L.: On the stability, or instability, of certain fluid motions. Proc. Lond. Math. Soc. 11, 57-70 (1879/80)

34. Routh, E.J.: Stability of a dynamical system with two independent motions. Proc. Lond. Math. Soc. 5, 97-100 (1873/74)

35. Routh, E.J.: On Laplace's three particles, with a supplement on the stability of steady motion. Proc. Lond. Math. Soc. 6 , 86-97 (1874/75)

36. Sharpe, F.R.: On the stability of the motion of a viscous liquid. Trans. Am. Math. Soc. 6(4), 496-503 (1905)

37. Chalishajar, D., Kumar, A.: Existence,uniqueness and Ulam's stability of solutions for a coupled system of fractional differential equations with integral boundary conditions. Mathematics 6(6), 96 (2018). https://doi.org/10.3390/math6060096 
38. Khan, A., Shah, K., Li, Y., Khan, T.S.: Ulam type stability for a coupled system of boundary value problems of nonlinear fractional differential equations. J. Funct. Spaces 2017, Article ID 3046013 (2017)

39. Xu, L., Dong, Q., Li, G.: Existence and Hyers-Ulam stability for three-point boundary value problems with Riemann-Liouville fractional derivatives and integrals. Adv. Differ. Equ. 2018, 458 (2018). https://doi.org/10.1186/s13662-018-1903-5

40. Sousa, J.V., Oliveira, E.C.: Ulam-Hyers stability of a nonlinear fractional Volterra integro-differential equation. Appl. Math. Lett. 81, 50-56 (2018)

41. Wang, J., LV, L., Zhou, Y.: Ulam stability and data dependence for fractional differential equations with Caputo derivative. Electron. J. Qual. Theory Differ. Equ. 2011, 63 (2011)

42. Berhail, A., Tabouche, N., Matar, M.M., Alzabut, J.: On nonlocal integral and derivative boundary value problem of nonlinear Hadamard Langevin equation with three different fractional orders. Bol. Soc. Mat. Mexicana 26, 303-318 (2020). https://doi.org/10.1007/s40590-019-00257-z

43. Ahmad, M., Zada, A., Alzabut, J.: Hyers-Ulam stability of a coupled system of fractional differential equations of Hilfer-Hadamard type. Demonstr. Math. 52(1), 283-295 (2019). https://doi.org/10.1515/dema-2019-0024

44. Zada, A., Waheed, H., Alzabut, J.: Existence and stability of impulsive coupled system of fractional integro-differential equations. Demonstr. Math. 52(1), 296-335 (2019). https://doi.org/10.1515/dema-2019-0035

45. Seemab, A., Rehman, M.U., Alzabut, J., Hamdi, A.: On the existence of positive solutions for generalized fractional boundary value problems. Bound. Value Probl. 2019, 186 (2019). https://doi.org/10.1186/s13661-019-01300-8

46. Matar, M.M., Amra, I.A., Alzabut, J.: Existence of solutions for tripled system of fractional differential equations involving cyclic permutation boundary conditions. Bound. Value Probl. 2020, 140 (2020). https://doi.org/10.1186/s13661-020-01437-x

47. Ali, Z., Zada, A., Shah, K.: Ulam stability to a toppled systems of nonlinear implicit fractional order boundary value problem. Bound. Value Probl. 2018, 175 (2018). https://doi.org/10.1186/s13661-018-1096-6

48. Waheed, H., Zada, A., Xu, J.: Well-posedness and Hyers-Ulam results for a class of impulsive fractional evolution equations. Math. Methods Appl. Sci. (2020). https://doi.org/10.1002/mma.6784

49. Zada, A., Pervaiz, B., Shah, S.O., Xu, J.: Stability analysis of first-order impulsive nonautonomous system on timescales. Math. Methods Appl. Sci. 43(8), 5097-5113 (2020). https://doi.org/10.1002/mma.6253

50. Rizwan, R., Zada, A.: Nonlinear impulsive Langevin equation with mixed derivatives. Math. Methods Appl. Sci. 43(1), 427-442 (2020). https://doi.org/10.1002/mma.5902

51. Zada, A., Fatima, S., Ali, Z., Xu, J., Cui, Y.: Stability results for a coupled system of impulsive fractional differential equations. Mathematics 7(10), 927 (2019). https://doi.org/10.3390/math7100927

52. Ahmad, M., Jiang, J., Zadaz, A., Shah, S.O., Xu, J.: Analysis of coupled system of implicit fractional differential equations involving Katugampola-Caputo fractional derivative. Complexity 2020, Article ID 9285686 (2020). https://doi.org/10.1155/2020/9285686

53. Ahmad, M., Zadaz, A., Wang, X.: Existence, uniqueness and stability of implicit switched coupled fractional differential equations of $\psi$-Hilfer type. Int. J. Nonlinear Sci. Numer. Simul. 21(3-4), 327-337 (2020) https://doi.org/10.1515/ijnsns-2018-0371

54. Amara, A.: Existence results for hybrid fractional differential equations with three-point boundary conditions. AIMS Math. 5(2), 1074-1078 (2019)

55. Niyom, S., Ntouyas, S.K., Laoprasittichok, S., Tariboon, J.: Boundary value problems with four orders of Riemann-Liouville fractional derivatives. Adv. Differ. Equ. 2016, 165 (2016) https://doi.org/10.1186/s13662-016-0897-0

56. Krasnoselskii, M.A.: Two remarks on the method of successive approximations. Usp. Mat. Nauk 10, 123-127 (1955)

57. Granas, A., Dugundji, J.: Fixed Point Theory. Springer, New York (2003)

\section{Submit your manuscript to a SpringerOpen ${ }^{\odot}$ journal and benefit from:}

- Convenient online submission

- Rigorous peer review

- Open access: articles freely available online

- High visibility within the field

- Retaining the copyright to your article

Submit your next manuscript at $\boldsymbol{s p r i n g e r o p e n . c o m ~}$ 